\title{
Rational Adaptation Under Task and Processing Constraints: Implications for Testing Theories of Cognition and Action
}

\author{
Andrew Howes \\ University of Manchester
}

\author{
Richard L. Lewis \\ University of Michigan
}

\author{
Alonso Vera \\ National Aeronautics and Space Administration Ames Research Center, Moffett Field, California
}

\begin{abstract}
The authors assume that individuals adapt rationally to a utility function given constraints imposed by their cognitive architecture and the local task environment. This assumption underlies a new approach to modeling and understanding cognition-cognitively bounded rational analysis - that sharpens the predictive acuity of general, integrated theories of cognition and action. Such theories provide the necessary computational means to explain the flexible nature of human behavior but in doing so introduce extreme degrees of freedom in accounting for data. The new approach narrows the space of predicted behaviors through analysis of the payoff achieved by alternative strategies, rather than through fitting strategies and theoretical parameters to data. It extends and complements established approaches, including computational cognitive architectures, rational analysis, optimal motor control, bounded rationality, and signal detection theory. The authors illustrate the approach with a reanalysis of an existing account of psychological refractory period (PRP) dual-task performance and the development and analysis of a new theory of ordered dual-task responses. These analyses yield several novel results, including a new understanding of the role of strategic variation in existing accounts of PRP and the first predictive, quantitative account showing how the details of ordered dual-task phenomena emerge from the rational control of a cognitive system subject to the combined constraints of internal variance, motor interference, and a response selection bottleneck.
\end{abstract}

Keywords: rational adaptation, bounded optimality, cognitive architecture, theory comparison, response ordering, dual task

The extraordinarily flexible and adaptive nature of human behavior presents both unique opportunities and unique challenges for developing a science of the mind and brain. On the one hand, treating the mind as an adaptive system opens up possibilities for deep explanations of behavior that are grounded primarily in the observable structure and contingencies of the task environment, along with an assumption of rationality or optimal adaptation. This

Andrew Howes, Manchester Business School, University of Manchester, Manchester, United Kingdom; Richard L. Lewis, Department of Psychology, University of Michigan; Alonso Vera, Human-Systems Integration Division, National Aeronautics and Space Administration (NASA) Ames Research Center, Moffett Field, California.

The work was supported by the NASA Aviation Operations Safety Program, by the NASA Intelligent Systems Program, and by the Office of Naval Research. We thank David Meyer and Eric Schumacher for providing the raw data for the experiments reported in Schumacher et al. (1999) as well as John Anderson, Nick Chater, Alina Chu, Wai Tat Fu, Wayne Gray, Collin Green, Bonnie John, Jonathon Kopecky, Stelios Lelis, Michael McCurdy, David Meyer, Hal Pashler, Stephen J. Payne, Roger Remington, Mason Smith, Yuan-Chi Tseng, and Richard M. Young for providing useful comments on this work.

Correspondence concerning this article should be addressed to Andrew Howes, Manchester Business School, MBS East, University of Manchester, Booth Street West, Manchester M15 6PB, United Kingdom. E-mail: Andrew.Howes@mbs.ac.uk insight is the point of departure for a range of approaches to understanding cognition and perception, including rational analysis and related Bayesian approaches (Anderson, 1990; Berthier, Rosenstein, \& Barto, 2005; Bogacz, Brown, Moehlis, Holmes, \& Cohen, 2006; Chater \& Oaksford, 1999; Geisler, 2003; Tenenbaum, Griffiths, \& Kemp, 2006), optimal motor control approaches (Maloney, Trommershäuser, \& Landy, 2007; Meyer, Abrams, Kornblum, Wright, \& Smith, 1988; Trommershäuser, Maloney, \& Landy, 2003a, 2003b; Reichle \& Laurent, 2006), as well as signal detection theory and ideal observer analysis (Green \& Swets, 1966; Swets, Tanner, \& Birdsall, 1961; Tanner \& Swets, 1954). For example, in the arena of perception, ideal observer models demonstrate that human performance on some very simple discrimination tasks is limited only by external photon noise (Geisler, 2003). In the arena of memory, the decay over time of items in human long-term declarative memory has been shown to correspond substantially to the decaying probabilities that the items will need to be selected given the statistical properties of the environment (Anderson, 1990).

On the other hand, the extreme flexibility of the human cognitive system means that any given task situation may be approached via an unbounded variety of strategies, making it very difficult to discern the invariant principles of cognition and action. It is often unclear whether empirical observations are due to invariant properties of the cognitive system or, instead, are due to strategic 
responses to the task. The signal detection theory (SDT) analysis of Tanner and Swets (1954) represents early recognition of the most elementary form of the problem: Even in the simplest possible perceptual experiments (detection), behavior is jointly determined by strategy (represented by the criterion variable level in SDT) and the noise inherent in the participants' perceptual representations and the external stimulus (represented by the $d^{\prime}$ measure). Indeed, Swets et al. (1961) were clear that the key contribution of SDT was its "separation of the factors that influence the observer's attitudes from those that influence his sensitivity" (p. 336).

A recent prominent example of the problem, taken up in detail below, is the explanation of slowing in certain dual-task situations: Is the slowing the result of an immutable property of the architecture, such as a selection bottleneck, or is it due to a strategy participants adopt (Meyer \& Kieras, 1997a, 1997b; Meyer \& Kieras, 1999)? In general, it can be difficult to discern the cognitive architecture - the fixed computational mechanisms that support cognition and coordinate perception and action (Anderson, 1983; Anderson et al., 2004; Newell \& Simon, 1972; Newell, 1990).

Following Howes and Young (1997), we refer to this problem as the architecture-strategy credit assignment problem. The problem is that, even with data obtained from rigorously designed experiments, it is often difficult to know whether to assign credit for the observed data patterns to some aspect of the cognitive architecture or to the strategies that people select in service of the task (Howes \& Young, 1997; Kieras \& Meyer, 2000; Meyer \& Kieras, 1997a). Observed effects may be a consequence of what people are trying to do, rather than of genuine constraints on what they can do. As a result, when researchers build computational models of the observed behavior, they often make (sometimes implicit) assumptions about the roles of strategy and architecture that cannot be supported by the data. Kieras and Meyer (2000) pointed out that for the most part researchers make informal assumptions about strategies. Rarely do they report or justify why they believe that participants are using one set of strategies rather than another. Instead strategies are chosen intuitively, often with the aim of maximizing the fit between a model and the data, so that empirical support for the architecture can be claimed. The model strategy is thereby used as a free parameter that is set with the aim of maximizing the credit assigned to the theory of the architecture.

Our goal for the present work is to specify and illustrate a new approach to developing and testing cognitive theories that explicitly deals with the large space of possible strategic responses to local task environments but exploits the adaptive nature of human cognition to sharply narrow this space to subsets that are rational given the local task demands. A distinguishing feature of our approach, which we call cognitively bounded rational (CBR) anal$y$ sis, is that it generates predictions from cognitive theory via strategies that are rationally selected given an explicit utility function (Howes, Lewis, \& Vera, 2007; Howes, Lewis, Vera, \& Richardson, 2005; Howes, Vera, Lewis, \& McCurdy, 2004; Lewis, Vera, \& Howes, 2004; Vera, Tollinger, Eng, Lewis, \& Howes, 2005; Vera, Howes, McCurdy, \& Lewis, 2004).

The approach has two related but distinct benefits. First, it allows us to pursue deep explanations of adaptive behavior by providing an answer to the question of why the behavior has the shape it does (the behavior is adaptive given the assumed archi- tectural constraints). Second, the approach increases our ability to discern the nature of the invariant internal mechanisms that bound the adaptation-it addresses the credit assignment problembecause it allows us to generate highly constrained predictions from a cognitive theory even when the space of possible adaptations is complex.

CBR analysis builds naturally on several lines of theoretical work in cognitive science. Specifically, it inherits from Simon's $(1955,1957,1991,1992)$ bounded rationality a focus on understanding the side conditions that bound adaptive behavior, but it differs in that it seeks to derive the optimal behavior given these constraints. It inherits from SDT the recognition (a) that all behavior is jointly determined by invariant properties of the cognitive system and by strategic factors, and (b) that psychological science requires empirical and analytic tools that take both factors into account. But it differs from SDT and analyses of optimal motor control (Maloney et al., 2007; Meyer et al., 1988; Trommershäuser et al., 2003a, 2003b; Reichle \& Lamant, 2006) in that it allow for analyses of a significantly broader class of tasks and provides a broader conception of strategic variation. It inherits from rational analysis (Anderson, 1990) a focus on understanding how behavior is adaptive. But it differs from rational analysis in that it focuses on strategic adaptation on shorter time scales in local task environments and explicitly takes into account a formal specification of cognitive architecture. Finally, the approach inherits from cognitive architecture research (Anderson et al., 2004; Meyer \& Kieras, 1999; Newell, 1990) an assumption of extreme strategic flexibility and a focus on specifying the task-invariant computational mechanisms of cognition and action, but it differs in that it moves beyond the simulation of single, intuitively generated strategies in favor of methods for deriving adaptive behavior given a potentially large space of possible strategies and an explicitly defined, taskspecific utility function.

An important benefit of the approach is that it addresses in a substantive way the concerns about model fitting expressed by Roberts and Pashler (2000) and the problems concerning strategic variation expressed by Kieras and Meyer (2000). When researchers use CBR analysis, models are calibrated on some aspect of the data, predictions are derived using the assumption of rational adaptation, and tests are made against the remaining data. Neither the model's architectural parameters nor its strategic parameters are adjusted to match the target data to be explained.

The principle domain of illustration in this article concerns understanding the bounds on dual-task performance (and their individual variation) in psychological refractory period (PRP) experiments, an issue of considerable theoretical importance in the development of theories of cognitive control and attention, and a topic of much recent debate (Anderson, Taatgen, \& Byrne, 2005; Byrne \& Anderson, 2001; Hazeltine, Teague, \& Ivry, 2002; Meyer \& Kieras, 1997a, 1997b; Pashler, 1998). The rationale behind PRP experiments was simple: The systematic slowing of responses that emerges as two separate choice-reaction tasks are brought into close temporal proximity was believed to provide insight into fundamental, architectural properties of human multitasking - the ability to focus thought on more than one thing at a time (for extensive historical overviews, see Meyer \& Kieras, 1997a; Pashler, 1998).

More specifically, the PRP debate has concerned the existence of a response selection bottleneck. Response selection bottleneck 
theories assert that there is a selection bottleneck that is a fixed part of the cognitive architecture, and this bottleneck permits cognition to be focused on only one task at a time. However, although the selection bottleneck account is appealing because it appears to provide an elegant explanation of a variety of PRP-related data patterns (Pashler, 1998), others, notably Meyer and Kieras (1997a), have pointed out that the same patterns could be due to strategic adaptation rather than to architectural limitations. The PRP paradigm therefore exemplifies the architecture-strategy credit assignment problem.

The plan for the remainder of this article is as follows. We first provide brief relevant background on related approaches in cognitive science that motivate our work, followed by an overview of the proposed approach. We then provide a concrete demonstration that current practice in applying theories of cognitive architecture does not take sufficient account of strategic variation. This demonstration takes the form of a detailed analysis of ACT-R (Anderson, 1993; Anderson \& Lebiere, 1998) models of PRP reaction times (Byrne \& Anderson, 2001) that shows that the attribution of explanatory credit to strategy or architecture is unclear for these models, and that therefore so is the resulting explanation of critical phenomena. We then describe our alternative modeling framework, CBR analysis. Next, we introduce a new theory of response ordering (such as that required in classic PRP tasks) called ordered response theory (ORT). The theory states that any two responses that must be ordered will be coordinated in a way that maximizes subjective expected utility. In standard tasks where both accuracy and speed are important, utility is increased by smaller temporal separations but decreased by reversal errors. ORT is consistent with the strategic-response deferment account of Meyer and Kieras (1997a, 1997b) but differs in the nature of the predictions and explanations that it provides: It yields quantitative predictions at the level of individual participants and accompanying explanations grounded in the assumption of rational adaptation.

So as to test ORT we introduce a set of auxiliary parametric assumptions about the processing architecture that are derived from EPIC (Meyer \& Kieras, 1997a, 1997b). We then specify a space of possible strategies for coordinating the two responses. The space is defined in terms of strategic parameters that govern the temporal separation of the responses. We then compare the prediction of the theory to data from four PRP experiments reported by Schumacher et al. (1999). This is accomplished by calculating the optimal strategy for each individual (using individually calibrated processing architectures) and using these optimal strategies to generate specific quantitative predictions at the individual level. The theory yields quantitative accounts of both aggregate and individual PRP curves and, arguably, a deeper explanation of PRP behavior than has been previously obtained. Furthermore, we show how it is possible to determine whether the predictions of the theory depend upon certain key architectural assumptions, such as the presence or absence of a cognitive bottleneck. Finally, in the General Discussion, we develop a set of general recommendations for cognitive modeling.

\section{Possible Responses to the Credit Assignment Problem}

\section{Rational Analysis}

The architecture-strategy credit assignment problem (Howes \& Young, 1997) is a special case of the identifiability problem in cognitive science: A theory of the architecture that is sufficiently powerful to exhibit the full range of human perceptual and cognitive capabilities is likely to be capable of mimicking the inputoutput characteristics of any other such candidate theory (Anderson, 1978; Pylyshyn, 1973). Rational analysis is one approach to the identifiability problem that seeks explanations in terms of environment (or at least its experience) and the goals of the cognitive system. Anderson (1990) stated a general principle of rationality: "The cognitive system operates at all times to optimize the adaptation of the behavior of the organism" (p. 28). Anderson (1990) started with the assumption that evolution has to some extent optimized cognition to its environment. He argued that within the limits set by what evolution can achieve, a species is at some stable point in time at a local maximum. Anderson (1990) proposed that if the principle of rationality were applied to the development of a theory of cognition, then substantial benefits would accrue. In particular, the rational approach (a) offers a way to avoid the identifiability problem, because the theory depends on the structure of an observable world and not on the unobservable structure in the head; (b) offers an explanation for why people behave the way they do rather than just for how they behave (because they gain benefit from optimization); and (c) offers guidance on the construction of a theory of the mechanism.

Researchers have used rational analysis to provide explanations of a number of phenomena that might otherwise be taken to indicate arbitrary constraints on cognitive mechanisms or limited rationality. For example, Oaksford and Chater (1994) argued that human performance on the Wason selection task (Wason, 1966) could be explained as optimal given a broader consideration of the probabilistic decision-making tasks encountered by people in their everyday lives. Anderson and Milson (1989) and Anderson and Schooler (1991) demonstrated how the decay of memory could be understood as functional given the rate of reduction in the utility of information in the world. They argued that if human memory decayed at the same rate as the utility of information in the world, then memory was optimally adapted and memory decay was rational. Similarly, Lovett and Anderson (1996) used rational analysis to develop a theory of how people select action based on their history of success.

Researchers have used some of these explanations to motivate theories of specific architectural mechanisms. In particular, Anderson modified $\mathrm{ACT}^{*}$ to reflect the insights gained from the rational analyses of memory and choice (Anderson \& Milson, 1989; Lovett \& Anderson, 1996). The resulting theory, ACT-R ( $\mathrm{R}$ is for rational), combined a model of the decay of activation in declarative memory, derived from the rational analysis of Anderson and Milson (1989), with a model of production rule conflict resolution derived from a rational analysis of the selection of action on the basis of history of success (Lovett \& Anderson, 1996). It is in this sense that rational analysis may achieve the goal of informing theories of mechanism: by providing abstract computational characterizations of the functions that components of the architecture should achieve, under certain assumptions about what they are adapted to.

Rational analysis thereby addresses the architecture-strategy credit assignment problem by focusing on the study of the task environment and assuming that (through evolution) it is the shape of this environment that has determined the architecture. As a consequence, and as Simon (1992) emphasized, rational analysis 
backgrounds the empirical study of locally adaptive behavior as a tool for determining the architecture.

\section{SDT and the Sperling and Dosher (1986) Generalizations}

Early in the study of perceptual decision making it was observed that regularities in the detectability of a stimulus could have as much to do with strategic adaptation as with the fundamental limits of the human architecture (Tanner \& Swets, 1954). SDT, which was developed as a consequence, is the earliest and perhaps best developed example of a formal analysis of human behavior that addresses strategic flexibility. The classic SDT task involves discrimination of a stimulus in which the presence of an auditory or visual stimulus must be detected against a background of noise. SDT assumes that people will select a detection threshold that is optimal given a utility function that trades the benefits of successful detections against the costs of false alarms. In general the noise that makes a stimulus hard to discriminate may be due to both external environmental noise and organism-internal noise. When the noise in a theoretical model is purely external, SDT yields ideal observer analyses: descriptions of the optimal performance of an observer unconstrained by internal limitations.

SDT thus shares with rational analysis the general dictum that appeal to internal processing limitations should be made only after explanations based on adaptation to the external environment have been found lacking. SDT and ideal observer analyses have been extremely influential and are widely used in psychophysics (e.g., Geisler, 2003).

Sperling and Dosher (1986) showed that a wide range of simple cognitive tasks are amenable to an SDT-like optimization analysis. These tasks go beyond standard SDT discrimination tasks and include choice reaction, visual search, and speed-accuracy tradeoff paradigms. The key generalization was to show that the continuous strategy space need not be restricted to discrimination thresholds but may also describe attentional resource allocation, assuming some internal limited processing resource. (A secondary generalization was to show that such continuous strategy spaces need not be uni-dimensional, though a multidimensional analysis was used for only one class of reaction-time task.) In short, Sperling and Dosher took an important step toward generalizing both the strategies and the internal constraints amenable to an optimization analysis. We believe the approach makes progress on the issue because the assumption that people are rational provides a means of narrowing the strategy space. This assumption and its consequence play a key role in CBR analysis as we develop in this article.

\section{Get Temporally Close to the Architecture}

Newell (1990) made explicit, and provided a solid theoretical basis for, a methodological dictum implicit in much empirical work in cognitive psychology: In order to see the architectural mechanisms clearly, one must get temporally close to them. The reason is that short time scales (at the level of hundreds of milliseconds to about a second, what Newell, 1990, termed immediate behavior) permit more limited opportunities for strategic variation-there are surely fewer ways for the human system to make a response to a stimulus in $500 \mathrm{~ms}$ than in $5,000 \mathrm{~ms}$.
The basic argument here is sound, but we believe that Newell (1990) underestimated the extent to which strategic variation is possible and present even in cases of immediate behavior-as the SDT analyses above might suggest. Strong evidence for this comes in the analyses and models presented by Meyer and Kieras (1997a, 1997b) in their account of PRP dual-tasking data, which demonstrated the possibility that the apparently clear empirical evidence for an architectural feature (the response selection bottleneck) was in fact consistent with a radically different architecture under alternative assumptions about strategy. The analyses presented later in this article further make this case.

\section{Use Empirical Methods That Provide More Direct Access to the Strategy}

This is the response urged by Newell and Simon (1972), who emphasized the use of verbal protocols, eye tracking, and other methods that would more directly reveal the intermediate states of cognition and so provide empirical evidence for the strategy used. These are surely useful techniques as demonstrated in Newell and Simon's landmark work on problem solving, but they are of limited applicability: They cannot be used to uncover strategies at the level of immediate behavior, the timescale where architectural mechanisms are most likely to show through. Thus, it is no surprise that Newell and Simon's theory of problem solving depended on, as they put it, "a few, and only a few, gross characteristics of the human [information processing system]" (p. 788). Rather, it was principally a theory of the strategies routinely adopted by humans to achieve boundedly rational behavior in problem solving. Newell and Simon were clear, however, that these strategies were shaped by the architecture, if only at a gross level. But there were no formal derivations of how different strategies might emerge from different architectural assumptions.

\section{Develop Theories of Instruction Taking and Task Acquisition}

Newell (1990; Lewis, Newell, \& Polk, 1989) also urged on psychology the development of theories of instruction taking and task acquisition. The benefits would be substantial: The formulation of specific task strategies would be taken out of the hands of the modeler and instead would be derived from possibly domaingeneral theories of instruction taking and learning. This is a laudable goal, and significant progress has been made toward it recently within the framework of the ACT-R theory (Anderson et al., 2005; Taatgen, 2005). However, the practical problem with this approach is that it puts an extremely difficult scientific problem for cognitive psychology (and artificial intelligence)—instruction taking - on the critical path toward making progress in identifying architectural mechanisms and strategies. We therefore believe we must pursue alternative parallel approaches in the meantime.

\section{Adopt More Principled Means of Comparing Data to Theories With Free Parameters}

One way to view strategic variation is that it introduces degrees of freedom in a model that can be used to fit data (though it is difficult to characterize quantitatively) (Lewis et al., 1989; Newell, 1990). Model fitting has recently come under much scrutiny 
(Kieras \& Meyer, 2000; Pitt, Kim, Navarro, \& Myung, 2006; Roberts \& Pashler, 2000), and it is worth considering whether recent methodological prescriptions for comparing data to parametric theories might be used to address the architecture-strategy credit assignment problem. Framed this way, the question is to what extent some given match to data provides support for the structural invariants of a theory with degrees of freedom.

Kieras and Meyer's (2000) concerns about strategic variation led them to propose the bracketing heuristic. In a bracketing analysis, the modeler determines both the fastest possible and the slowest reasonable strategy, and human performance is compared to see if it falls within the bracketed range. The degrees of freedom of the model are thereby exposed, and fitting can be avoided. For an example of the use of bracketing, see Gray and Boehm-Davis (2000).

Roberts and Pashler (2000) argued that, in general, the practice of fitting theories to data, for which strategy sampling is one technique, provides no empirical support for the theory. They asserted that a good fit between a model and the data reveals nothing about (a) how much the theory cannot fit, (b) how firmly the data rule out what the theory cannot fit, or (c) whether the theory could have fit any plausible result. Roberts and Pashler suggested that a better way to test a theory is first to determine the full range of a theory's predictions, then to determine how firmly the data are consistent with this range, and lastly determine whether plausible alternative outcomes are ruled out by the theory. These suggestions have helped shaped the analyses presented in this article.

A key element of the approaches adopted by Roberts and Pashler (2000) and by Kieras and Meyer (2000) derives from the recognition that a theory of cognition often predicts a space of behaviors, rather than a particular behavior, and that theory testing involves exploring this space. However, the space of possible strategies is large and, as one shall see, a theory of the architecture may as a consequence predict rather little. In these circumstances, an approach to limiting the range of predictions is required.

\section{An Analysis of Byrne and Anderson's (2001) Models of PRP Effects}

Before describing CBR analysis, we first present an analysis of some of the best existing cognitive architecture models of PRP dual tasking, which makes clear both the advantages and the weaknesses of current methods of modeling. The data and task discussed in this section will then be carried through the article as the primary example used to illustrate the new approach.

\section{The Theoretical Significance of PRP Phenomena}

The paradigmatic PRP data pattern, shown in Figure 1, arises when participants are instructed to respond to both tasks as quickly as possible but are asked to respond to Task 1 first. Task 2 response times are generally unaffected at long stimulus onset asynchronies (SOAs), the time between the first and second task stimuli, but then begin to increase monotonically at short SOAs. The empirical shape of the increasing part of the curve (often referred to as the PRP effect) and its theoretical interpretation have been the subject of much debate (see Meyer \& Kieras, 1997a; Pashler, 1998, for a review); we provide only a summary of the



Figure 1. The paradigmatic relationship between stimulus onset asynchrony (SOA) and response time (RT) in the psychological refractory period task paradigm. At short SOA, participants prioritize Task 1, and the time required to respond to Task 2 is increased.

relevant aspects that will allow us to frame the specific contributions made by the CBR analyses to follow.

\section{An Explanation in Terms of Strategic Processing}

The response selection bottleneck explanation of PRP phenomena was questioned by Meyer and Kieras (1997a, 1997b), who provided detailed critiques of the selection bottleneck assumption on both theoretical and empirical grounds. The centerpiece of their criticism was a set of computational models in EPIC that abandoned a selection bottleneck in favor of full cognitive parallelism and accounted for classic PRP effects via strategic processing. Their strategic response deferment models assert that people strategically defer Task 2 responses in order to conform to the explicit instructions, reinforced by the payoff regime, to give priority to Task 1. The issue remains controversial, as other studies indicate the presence of a selection bottleneck when strategic concerns are investigated with changes to the instructions (e.g., Levy \& Pashler, 2001; Ruthruff, Pashler, \& Klaassen, 2001) or when tasks involve substantial cognitive processing requirements (Byrne \& Anderson, 2001).

The EPIC work embodies two central claims concerning selection bottlenecks:

(a) The first, weaker claim is that the bottleneck assumption is not required to explain classic PRP effects; it is possible to obtain excellent quantitative fits using a parallel model with sufficient strategic variability. This result is a negative one in that it makes clear the existence of a form of the identifiability problem: The PRP data do not uniquely reveal the outlines of a serial architecture. 
(b) The second, stronger claim is that various detailed aspects of PRP data are consistent only with a strategic parallel model and rule out the serial bottleneck theory. These aspects include the subadditivity of cognitive complexity effects observed when two versions of Task 2 are used that differ in the difficulty of the response mapping (e.g., Schumacher et al., 1999). We discuss these subadditivity effects below.

We shall now briefly consider responses to these claims and the specific role that cognitive architecture models play in these responses.

\section{The Architecture-Strategy Credit Assignment Problem in Explaining PRP Phenomena}

The counterargument to the first claim is that the serial bottleneck model is to be preferred on the basis of parsimony. More specifically, so this line of argument goes, because EPIC does admit to arbitrary strategic flexibility, it could be used to fit almost any pattern of data, including patterns that are empirically unobserved, unlike the simple bottleneck model, which makes unambiguous predictions of qualitative data patterns that are observed, at least in the classic PRP paradigms. Thus, according to the logic of Roberts and Pashler (2000) (which we consider in more detail below), the serial bottleneck theory receives strong support from PRP data, while the EPIC theory receives no support, despite the demonstration that the data are consistent with a parallel architecture.

The major problem with this argument, alluded to in the introduction above, is that systematically abiding by its methodological implications will always result in the rejection of strategic variability as an explanation because it significantly introduces additional theoretical degrees of freedom. Under current modeling practice, it is usually the case that for any given cognitive phenomenon a strategic explanation will suffer under such parsimony comparisons to a competing local theory with minimal, structural assumptions (assuming that the strategies cannot be directly observed). But given the overwhelming a priori reasons to believe that the human system does in fact have such adaptive flexibility, adopting the nonstrategic stance for each local cognitive phenomenon to be explained significantly increases the risk that phenomena will be misinterpreted as providing information about fixed cognitive mechanisms when in fact they are revealing of the strategies that participants adopt (Newell, 1973, 1990; Pylyshyn, 1973).

A secondary problem with the strong form of this argument is that it claims that EPIC could reproduce any PRP data pattern, but this has not been demonstrated nor is it obviously true. Addressing this issue imposes a significant modeling burden, and we believe a substantive response demands the kind of framework we are proposing in this article, one that permits more systematic largescale simulations of spaces of strategies than have been previously attempted.

\section{A Selection Bottleneck Architecture With Strategic Flexibility}

The counterargument to the second, stronger claim-that certain PRP data patterns are inconsistent with a serial bottle- neck theory-is provided by computational models constructed in the ACT-R architecture. ACT-R is a production system architecture that permits strategic flexibility as EPIC does but that embodies a selection bottleneck. ACT-R serial bottleneck models have been provided for dual-task data that have been previously assumed to be problematic for a serial bottleneck theory. Most notably, these include the Byrne and Anderson (2001) models of Schumacher et al.'s (1999) PRP data that give accounts of the subadditive effects of response mapping difficulty and recent serial bottleneck models (Anderson et al., 2005) of Hazeltine et al.'s (2002) perfect time-sharing data.

It is important to understand that the original Meyer and Kieras (1997a) criticisms of selection bottleneck theory were criticisms of a verbally stated model with no locus for strategic adaptation. The ACT-R bottleneck account, in contrast, is a computationally explicit model that generates quantitative predictions and does allow for strategic variability. Thus, the ACT-R models are subject to the same criticisms above concerning the degrees of freedom introduced by strategic variation-the serial model as embodied by ACT-R is comparable to EPIC in this regard. Where does this leave us, then, with respect to conclusions that we can safely draw concerning theoretical explanations of the classic PRP effects?

\section{Parallel and Serial Cognitive Architecture Accounts of PRP: What Can We Conclude?}

For present purposes, we summarize the current state of affairs with respect to the classic PRP effect as follows (we consider the implications of our approach for related paradigms in the General Discussion). The EPIC models appear to demonstrate that parallel models with strategic variation are consistent with a wide range of existing PRP data, including some potentially problematic for the classic selection bottleneck accounts. The ACT-R models appear to demonstrate that serial models with strategic variation are consistent with a range of PRP data, also including some data thought to be problematic for the classic selection bottleneck accounts. There are compelling reasons for adopting strategically flexible computational models (outlined above), and these models and accompanying detailed analyses of the data represent significant progress in understanding PRP phenomena beyond that provided by the simple bottleneck theory. But despite this, we also argue that, in the bargain, a deeper explanation of the data has potentially been lost. We say "potentially been lost" because the range of possible predictions of the serial and parallel theories has not yet been made explicit, and we do not have clear independent justifications for the specific strategies adopted in these accounts. We do not therefore know whether, as claimed, the theories predict observed PRP effects or whether a consequence of the degrees of freedom associated with strategic flexibility is that the theories could be fit to almost any effect. As we now demonstrate, applying the techniques of CBR analysis to these problems can address both of these concerns and can significantly clarify what has, and has not, been learned from the data and models.

In the next part of the article, we introduce Schumacher et al.'s (1999) PRP tasks and then report a detailed analysis of the contributions of architecture and strategy to Byrne and Anderson's (2001) models of Schumacher et al.'s (1999) experiments. Our aim is to demonstrate how difficult it is to argue that architectures that permit strategic flexibility (such as EPIC and ACT-R) explain data 
based on evidence of a good fit, in the absence of a clear rational basis for the selected strategy.

In the second main part of the article, we present the ORT and accompanying CBR analyses of the Schumacher et al. (1999) PRP tasks. The models select specific strategies based on their adaptiveness to the task (as measured objectively by the specific payoffs used in the Schumacher et al., 1999, experiments), not on the quality of fit between model predictions and data. Nevertheless, the correspondence between the data and the resulting quantitative predictions provides compelling evidence that participants in these experiments adapted optimally or nearly optimally to the combined constraints of the task environment and their own cognitive architectures - in particular, noise and modality-specific response preparation bottlenecks. Through analyses of individual participant data, we furthermore show that the dual-task effects (as represented in the PRP curve) vary considerably across participants but that this variation may be accounted for as a rational adaptation to individually varying architectural constraints. Finally, through modeling experiments that test specific bottleneck assumptions, we demonstrate that the PRP tasks provide strong evidence for modality-specific response preparation bottlenecks but much weaker evidence for response selection bottlenecks.

\section{Schumacher et al.'s (1999) PRP Experiments}

Schumacher et al. (1999) reported a series of experiments in the classic PRP paradigm. The third experiment will serve to illustrate the basic structure. In this experiment participants were required to respond to a tone (Task 1) and either a hard or an easy visual classification task (Task 2) with keypresses that depended on whether the tone was high or low and whether the pattern contained a particular feature. The hard version of Task 2 used an incompatible mapping from stimulus to response, and the easy version used a compatible mapping. The tone and the pattern were presented with a gap of between 50 and 1,000 ms (SOA). Participants were asked to prioritize Task 1 (i.e., to respond to the tone first and avoid response reversals). The Task 1 response times (RT1s) were, on mean, unaffected by SOA. In contrast, the mean Task 2 response time (RT2), at a short SOA (50 ms), was greater than the RT2s at long SOAs but less than the sum of RT1s and RT2s at long SOAs (>500 ms). Clearly some parallelization is possible but, in addition, something causes the Task 2 response to slow.

Following Meyer and Kieras (1997a), Schumacher et al. (1999) examined the subadditivity of the Task 2 difficulty effect. Recall that subadditivity is the difference between the difficulty effect at long SOA and the difficulty effect at short SOA. Meyer and Kieras (1997a) had used the presence of subadditivity as evidence against a selection bottleneck. They reasoned that if the delay in RT2 at short SOAs is entirely due to a response selection bottleneck, then no subadditivity would be expected (i.e., the difficulty effect at short SOA should be the same as at long SOA). If, in contrast, the delay in RT2 at short SOAs is due, at least in part, to a strategic postponement of the Task 2 response, then subadditivity should be observed (i.e., the difficulty effect at short SOA should be less than the difficulty effect at long SOA). Strategic response deferment creates slack time between Task 2 response selection and Task 2 motor processing into which task difficulty can be absorbed. (The logic depends on the assumption that the effect of task difficulty is to increase the time required for response selection processing and not perceptual-motor processing.)

The four experiments reported by Schumacher et al. (1999) vary in the nature of the difficulty manipulation and the modalities of the stimulus-response pairings. The experiments are referred to in the rest of this article as E1,E2,E3, and E4. E1 Task 1 was a two-choice visual-manual task, and Task 2 was an auditorymanual task. Difficulty was manipulated with the number of stimulus-response mappings. E2 Task 1 was an auditory-manual task, and Task 2 was a visual-manual task. In E3 (described above), Task 1 was an auditory-manual task similar to that used in E2, and Task 2 was a visual-manual task; difficulty was manipulated with stimulus-response compatibility. In E4, Task 1 was auditory-vocal, and Task 2 was visual-manual; again, difficulty was manipulated with stimulus-response compatibility.

Importantly, participants in all four of Schumacher et al.'s (1999) experiments were rewarded monetarily according to a quantitative payoff scheme, and they received instruction on the nature of this payoff and feedback on their progress throughout the experiment. We refer to a payoff scheme as an objective utility function. Although there were small differences in the schemes, a successful pair of responses was rewarded with 100 points minus 1 point for every $10 \mathrm{~ms}$ taken to respond. Response reversals and incorrect responses were given minus 100 points. Extra points were given if short-SOA Task 1 performance was within $75 \mathrm{~ms}$ of long-SOA Task 1 performance (an attempt to prevent response grouping). Thus, a precise speed-accuracy tradeoff was imposed upon the participants.

\section{An Analysis of the Role of Strategy in an Existing Account of PRP Effects}

Byrne and Anderson (2001) reported detailed ACT-R models of Schumacher et al.'s (1999) E1-E4 that generated reaction time predictions for each combination of SOA, task, and Task 2 difficulty. For all four models, Byrne and Anderson estimated parameters based on single-task or long-SOA performance only and predicted short-SOA, dual-task RTs without further quantitative parameter estimation. They also reused values estimated for models of one experiment for models of other experiments (e.g., the duration of the perceive process in E3 is reused in the model of E4). Byrne and Anderson (2001) achieved good fits between models and aggregate $\mathrm{RT}$ data, with $R^{2}$ values ranging from 0.92 to 0.99 and root-mean-squared errors (RMSEs) ranging from 10 to $19 \mathrm{~ms}$.

These ACT-R models combine several desirable features: (a) They obtained good quantitative fits, as reported above; (b) they reproduced key patterns of the data that are thought to have important theoretical implications (these include the basic PRP curve and the subadditive difficulty effect); and (c) they obtained these fits by reusing some parameter values across experiments, and fitting parameters based only on a limited subset of conditions (the long-SOA conditions) and using these fixed parameter values to generate predictions for the remaining conditions. In short, the models apparently embody claims of invariant quantitative relationships that should hold in the empirical data; the fact that these quantitative relationships are observed is thus an impressive accomplishment of the modeling. 
Using models based on a general cognitive architecture such as ACT-R to account for dual-task phenomena holds the promise of providing more principled explanations than would otherwise be possible, because the independently motivated and fixed structure of the architecture is not modified in order to accommodate the data (Newell, 1973, 1990). But in order to fully understand the nature of the predictions and explanations such models provide, we must understand how they depend upon both the fixed architectural constraints and the strategies that are programmed into the architecture to perform the task. Arriving at such an understanding requires an analysis that makes explicit the space of plausible strategies for the architecture and the range of predictions corresponding to this space. Our immediate aim in undertaking this analysis is to provide a concrete illustration of the potential problems that arise when architecture is constrained in a principled fashion, but strategy is not.

\section{Overview of the Analysis}

The main idea behind our analysis of the Byrne and Anderson (2001) models is the following. The specific ACT-R models reported as providing good fits to the PRP data in Schumacher et al.'s (1991) E1-E4 embody a detailed set of specific strategic choices about how to coordinate the two tasks. These strategic choice implicitly define a space of possible strategies; we formalize a subset of this space in a systematic way and generate the predictions of the architecture across this space. We can then compare the full predictive range to human data and begin to understand the relative role of architecture and strategy in accounting for the phenomena.

To accomplish this analysis, we reconstructed the ACT-R models using a tool called CORE (constraint-based optimizing reasoning engine; Howes et al., 2004, 2005, 2007; Vera, Howes, McCurdy, \& Lewis, 2004). CORE was used to reconstruct both the relevant aspects of the ACT-R functional architecture and the strategy space required to perform the PRP task. It would be possible in principle to perform such an analysis using ACT-R itself, but CORE has the virtue of providing explicit support for compact generative representations of strategy spaces and a more transparent and explicit specification of the key architectural constraints. We now summarize briefly the architectural and strategy assumptions; further details are provided in the Appendix.

\section{Architectural Assumptions}

The key assumptions are (a) a serial cognitive processor that works in parallel with a set of perceptual-motor processors (cf. Meyer \& Kieras, 1997a), (b) motor processor preparation times dependent upon the number of features to be set in the motor system (following Meyer \& Kieras, 1997a), and (c) response retrieval times dependent upon the activation of chunks in memory. In the ACT-R theory, activation is dependent on usage history, but in Byrne and Anderson's (2001) models it is a parameter that is set so as to fit retrieval time at long SOA.

The consequences of these three assumptions can be illustrated graphically with a cascade graph $^{1}$ (see Figure 2). Figure 2 depicts a model of PRP behavior at an SOA of $50 \mathrm{~ms}$. The figure is a graph with resources (information processors) on the vertical axis and time on the horizontal axis. Processes, executed by processors, are represented with horizontal boxes. The left end of a process represents its start time, and the right end represents its end time. Information flows between processes are represented by vertical, connecting bars. Light-shaded processes represent Task 1, and dark-shaded processes represent Task 2 . The serial cognitive processor operates in parallel with the perceptual-motor processors. The duration of both the motor process and the retrieval time is contingent on the task.

\section{Strategy Assumptions}

The task knowledge for each strategy consisted of knowledge for how to achieve Task 1 and knowledge for how to achieve Task 2 in the context of Task 1. Rather than explore the individual instances of strategies used by Byrne and Anderson (2001), we specified a strategy space constructed from three orthogonal dimensions that capture key aspects of the strategic variability implicit in the ACT-R models. The space represents different ways of coordinating Task 1 and Task 2 performance by making certain aspects of Task 2 processing contingent upon certain aspects of Task 1 processing. The space thus generates strategies in the class of strategic response deferment models (Meyer \& Kieras, 1997a). The dimensions of this strategy space are as follows:

(a) Task 1 completion control signal. This signal can be thought of as a flag that is posted in working memory when Task 1 is viewed as sufficiently complete for Task 2 to proceed. There were two levels of Task 1 completion control signal. The ACT-R motor system represents two control states that are available for inspection by the cognitive production rule system. The first is after the prepare process and is called preparefree. The second is after the "init" process and is called processorfree. We explored the implications of deferring the Task 2 response until after each of these events.

(b) Explicit unlock process. If Task 2 has been suspended while Task 1 completes, then an unlock process subsequently allows Task 2 to proceed at the appropriate time. There were three levels of unlocking. We explored strategies that (a) deferred Task 2 with an unlock process, (b) strategies that had no unlock and did not defer Task 2, and (c) strategies that deferred Task 2 but without an unlock. If a strategy included an unlock, then it deferred processing of Task 2 response transmission.

(c) Task 2 attend deferment. An attend process directs the perceptual system to gather information. There were two levels of deferment of the Task 2 attend operator (for visual or auditory input). The attend of Task 2 was either deferred until after the response transmission of Task 1 or it was not.

This space yields 12 strategies $(2 \times 3 \times 2)$. We used CORE to automatically generate models of the same 12 strategies for each of the 2 conditions of the 4 experiments (96 models in total). Definitions of each of the strategies are given in the Appendix.

\footnotetext{
${ }^{1}$ Cascade graphs are a variant of the Gantt-style charts introduced in Gray, John, and Atwood (1993) and used by Gray and Boehm-Davis (2000) to depict CPM-GOMS models. Cascade graphs differ from CPMGOMS charts in that information flow dependencies between processes are not permitted unless the processes overlap in time (hence forming a cascade of information flow). This forces the model to be explicit about the cognitive resources used to buffer or transmit information between temporally distal processes (Howes et al., 2005).
} 


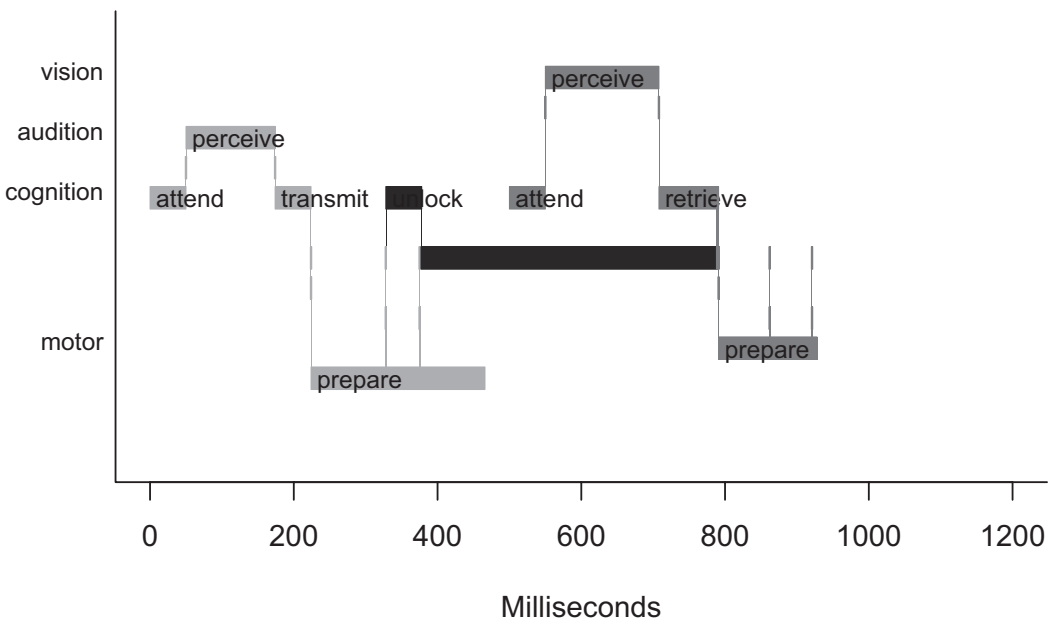

Figure 2. Assumptions concerning the architectural resources (vision, audition, etc.), processes (attend, perceive, etc.), and control information required to perform a psychological refractory period trial are represented here with a cascade graph. Information flows from one process to another are represented with vertical lines. Motor response to Task 2 is deferred until after the prepare stage of Task 1 using the unlock process.

\section{Analysis Results}

Following Byrne and Anderson (2001), we selected parameters so as to fit to long-SOA performance and we then used the models to predict short-SOA performance. We used a Monte Carlo algorithm to estimate the RT effects corresponding to each strategy for each experiment. Each of the models was run for 10,000 trials $(2,000$ trials for each level of SOA), sufficient to obtain $95 \%$ confidence intervals of less than $5 \mathrm{~ms}$ around the predicted means.

In what follows, we report the results of the simulations for RT1s and RT2s, paying special attention to the subadditive difficulty effects in Task 2 . We calculated confidence intervals around the human data at each level of SOA using the betweenparticipants variance of the raw data from the experiments reported in Schumacher et al. (1999). ${ }^{2}$ The raw data from Schumacher et al. (1999) were used for all analyses presented in this article.

For RT1s, all predictions corresponded closely to the data for both easy and hard conditions of all four experiments. This is not surprising given the success of Schumacher et al. (1999) in motivating most of their experiment participants to produce essentially flat RT1s. ${ }^{3}$ We thus focus all remaining analyses on RT2s, where the interesting PRP and subadditivity effects arise.

\section{Predictions for RT2s}

Plots of RT2s against SOA are shown in Figure 3. There are four pairs of panels, one pair for each experiment. Left panels represent performance on easy conditions, and right panels represent performance on hard conditions. The solid lines represent the RT2 means of the human data. The dashed lines represent the model predictions. Note that there are 12 dashed lines in each panel, one for each of the specific identified strategies (see the Appendix).

The fit of the models to 1,000-ms SOA is evident in each model. All 12 strategies generate similar performance at 1,000-ms SOA, but predicted RT2 can diverge significantly below 500-ms SOA. At 50-ms SOA there is substantial divergence in the predictions of each strategy in both conditions of all four experiments. For example, in E4 hard, divergence between predicted performances of each strategy is from below $500 \mathrm{~ms}$ to above $700 \mathrm{~ms}$ (see Figure 3, E4 hard).

For some of the strategies, across all four experiments, the data fall outside the confidence interval. The models appear noticeably better for some conditions (e.g., E2 easy) and noticeably worse for others (e.g., E3 hard). Some strategies predict faster performance than were observed, and some strategies predict slower performance.

It is also apparent that some of the strategies would have been consistent with data in which SOA has no effect. For example, this is true in E2 hard, E3 hard, E4 easy, and E4 hard.

\section{Predictions of Subadditivity Effects}

Given the importance of the subadditivity difficulty effects in the theoretical interpretation of the PRP effects, we also explored whether the subadditivity is an invariant prediction of the ACT-R models across the strategy space. To determine this, we generated predictions of subadditivity effects by subtracting the difficulty effects predicted by each pair of the 12 easy and 12 hard strategies - all of the possible ways of combining strategies for easy and hard tasks. There were a total of $12 \times 12=144$ subadditivity predictions.

The result of this analysis indicates that ACT-R can predict both the presence and the absence of subadditivity. The range of predicted subadditivity effects for $\mathrm{E} 4$ was from $-9 \mathrm{~ms}$ to $84 \mathrm{~ms}$. This range encompasses the data, but it also encompasses the prediction

\footnotetext{
${ }^{2}$ We are grateful to Eric Schumacher and David Meyer for providing us with the raw data.

${ }^{3}$ No participants were removed, although we know from later analyses that some failed to achieve flat RT1. Participants were not removed because failure to achieve flat RT1s is not evident in the means as used by Byrne and Anderson (2001).
} 

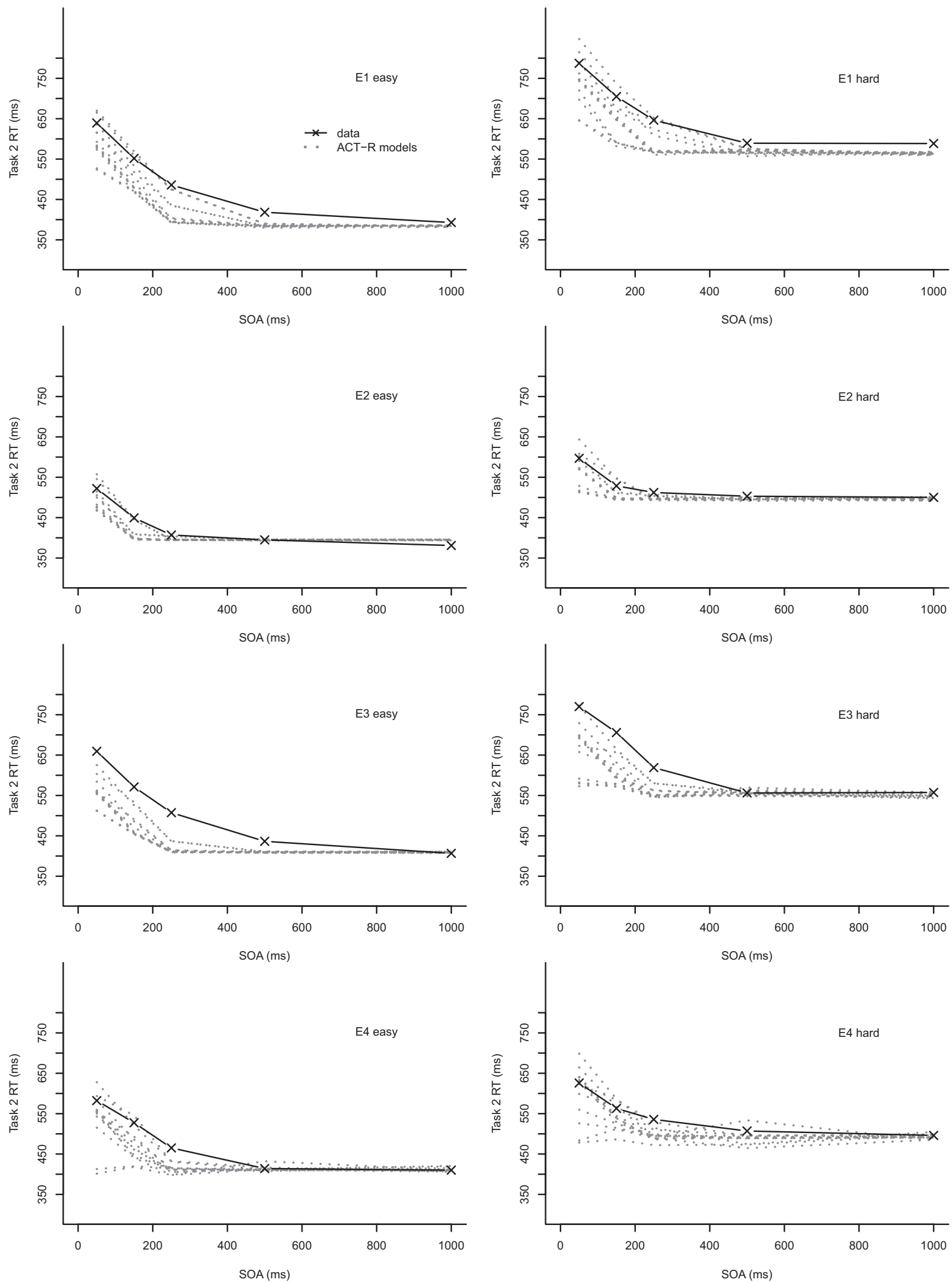

Figure 3. Task 2 response time (RT) against stimulus onset asynchrony (SOA) for data and ACT-R models based on those reported by Byrne and Anderson (2001). The data are reanalyzed from Schumacher et al.'s (1999) Experiments (E) 1-4. 
that there will be no subadditivity. Similarly broad ranges of subadditivity were obtained for E1, E2, and E3.

\section{Summary of the Analysis of the ACT-R Models}

We conducted a systematic analysis of the range of RT predictions made by the ACT-R architecture for performance on four PRP experiments reported by Schumacher et al. (1999). The analysis was conducted with the aim of providing a concrete illustration of the role that strategy may play in the application of architectural theory. The analysis determined the predictions of the architecture given a space of plausible strategies rather than the predictions of the architecture plus an intuitively selected strategy that provides a good fit to observed data. The strategy space that we formalized in the models was a subspace of the space implicitly explored by Byrne and Anderson (2001) and Meyer and Kieras (1997a, 1997b).

Over this strategy space, our reconstruction of the ACT-R models generated dual-task effects that sometimes correspond to the human data and sometimes do not. The models also predicted both the presence and the absence of subadditive difficulty effects for Task 2. With respect to the architecture-strategy credit assignment problem, this analysis therefore strongly suggests that it is the specific strategies programmed by Byrne and Anderson (2001) that yield the good fits, not the architecture itself. But the basis for the use of these particular strategies, rather than others that do not fit the data, is unclear. We therefore believe that, despite the fact that Byrne and Anderson used a constrained architecture with default values for some parameters and calibrated other parameters such as base-level activation to $1,000-\mathrm{ms}$ SOA RTs, it is not possible to assert that the ACT-R architecture explains or predicts the data on the basis of the reported analyses.

But Byrne and Anderson's (2001) primary objective in constructing the models was to demonstrate that a selection bottleneck theory (such as ACT-R) is consistent with the PRP data, including the subadditive difficulty effect, and that therefore these data and the subadditivity effects do not discriminate between bottleneck and no-bottleneck models (in contrast to the claims of Schumacher et al., 2001). The primary objective was not therefore to provide explanations of detailed quantitative data (though this claim was in fact made in Byrne \& Anderson, 2001; see pp. 851, 853, 854, and 868). In light of the preceding analysis, we can ask the following: Has the aim of consistency proof been realized?

Presumably a consistency proof carries with it weaker constraints on how theoretical degrees of freedom (whether quantitative parameter settings or strategy choices) are fixed in order to match data. The issue at hand is how weak the constraints are: Should the analyst be given unlimited license in selecting a strategy to match the data for the purpose of a consistency demonstration? We believe the answer is no and that it is ultimately more informative in the present case to ask, is ACT-R sufficient to account for subadditivity effects, assuming that people select rational strategies? We briefly explain our reasoning here because it helps to clarify the larger concern about the role of strategy in architectural modeling.

Consider both possible outcomes of a consistency demonstration that does not demand the rational selection of strategies. Suppose that the result were negative- - that is, suppose the models did not successfully match the data or produce the subadditive effect. It should be clear that no strong conclusions about architecture could be drawn from a single negative model result; it leaves open the possibility that a different strategy might have fit the data without changing the critical architectural feature in question (the bottleneck). It is impossible to know this without the systematic evaluation of some reasonable baseline strategy space.

Now consider in more detail the (actual) positive outcome: A particular strategy did yield reasonable fits to the data and did produce a subadditive difficulty effect. But this result naturally raises the question: why this particular strategy and not others? Was this strategy a rational and plausible one for the task? Were there other strategies that, if adopted, would provide significantly greater payoff for the participant but which do not actually match the data? Alternatively, is the critical prediction (here, subadditivity) invariant against strategy choice the strongest possible result?

In our view, it is not possible to appropriately assess either negative or positive outcomes of a consistency demonstration in the absence of a systematic evaluation of some baseline strategy space. And the evaluation of the strategies should be not only in terms of their match to data but also in terms of their rationality, which may be assessed by adopting a plausible subjective utility function and computing the payoff for each strategy. This is the essential idea behind the new approach that we now describe in detail.

\section{A General Approach to Understanding Adaptation to Task and Architecture}

The analysis presented in the previous section provides a concrete demonstration of the importance of exploring the space of possible adaptations - the space of strategies - in order to determine the relative contribution of cognitive architecture and strategy to prediction. We now describe the general approach, CBR analysis, that combines this strategic exploration with an evaluation of the utility of the strategies. A useful way of understanding $\mathrm{CBR}$ analysis and related approaches is to distinguish the types of rationality on which they are based. We identify four types of rationality: $\mathrm{N}, \mathrm{A}, \mathrm{B}$, and $\mathrm{C}$, each of which can be treated as a substantive hypothesis about the nature of human cognition:

1. Type $\mathrm{N}$ rationality is normative rationality. It identifies the optimal behavior given a local external task environment and assumed local utility, without consideration of psychological constraints or the broader time scales and contexts (e.g., lifetime or evolutionary) in which the utility of behavior may be evaluated.

2. Type A rationality is architectural rationality. People are Type A rational if the human cognitive architecture is itself optimally adapted to the evolutionary task environment. Rational analysis (Anderson, 1990) introduces the idea that people are Type A rational. A Type A rational architecture generates adaptive behaviors for the tasks for which human evolution has equipped it.

3. Type B rationality is bounded rationality. Individuals are Type B rational if they find behaviors that are sufficient in local task environments (Simon, 1992, 1955). Type B rational behaviors are sufficient to achieve objectives given limits set by the cognitive architecture and by the 
task environment, but they may well not be optimal under the (possibly implicit) utility function that defines what is sufficient. Simon (1955) articulated how aspiration levels may change in response to feedback over trials but did not offer a view on whether this (learning) process will lead to a behavior that is optimal given the constraints.

4. Type $\mathrm{C}$ rationality is cognitively bounded rationality. A system is Type $\mathrm{C}$ rational if its behavior maximizes subjective expected utility given the constraints on the cognitive architecture and the local task environment. If people are Type $\mathrm{C}$ rational with respect to a particular task, then they are boundedly rational (Type B) in the sense that performance is subject to limits (Simon, 1992), but in addition and with feedback over repeated trials, they maximize subjective utility given these limits. Type $\mathrm{A}$ and Type $\mathrm{C}$ rationality are orthogonal hypotheses; Type $\mathrm{C}$ rational behavior may or may not be constrained by a Type A rational architecture.

A CBR analysis is defined as any method that derives Type C rational behavior. We now describe our initial candidate method for performing such analyses.

\section{Overview of Each Step in the Method}

Figure 4 provides a high level overview of the method. The labels (1-5) in the figure describe the basic steps, and the circles illustrate how each step narrows an implicit space of possible behaviors until finally a space of behaviors emerges that constitutes the theoretical predictions to be compared to data. Step 1, specifying the environment and architecture, implicitly specifies a (large) space of possible behaviors. Step 2, specifying the strategy space, narrows the implicit space of possible behaviors to a specific subset of the intersection of those behaviors permitted by the external task situation and those behaviors architecturally possible. (Computing this intersection can be profitably viewed as a constraint satisfaction problem; Howes et al., 2004; Vera et al., 2004.) Step 3, inferring utility, computes the payoff for behaviors in this space with respect to an explicit utility function. Step 4, selecting the best strategies, identifies the much narrower subspace of behaviors that maximizes the utility function. Under a CBR analysis, this subspace of behaviors is jointly a function of the cognitive architecture and task constraints expressed as a space of possible strategies. The quantitative utility function defines what is best: It serves to select those strategies that-given the architecture-yield optimal behaviors. In Step 5, compare to data, we can then ask whether these optimal behaviors are what is actually observed in the given task situation. CBR analysis commits to the idea that through practice people implicitly work toward maximizing utility functions, and that for a cognitive theory to explain behavior the optimal behavior implied by the theory must show substantial correspondence to the asymptote bounding observed human performance.

We now describe these steps in further detail. For each step we identify an abstract set of requirements that the step places on both the human empirical paradigms and formal computational techniques that support the method.

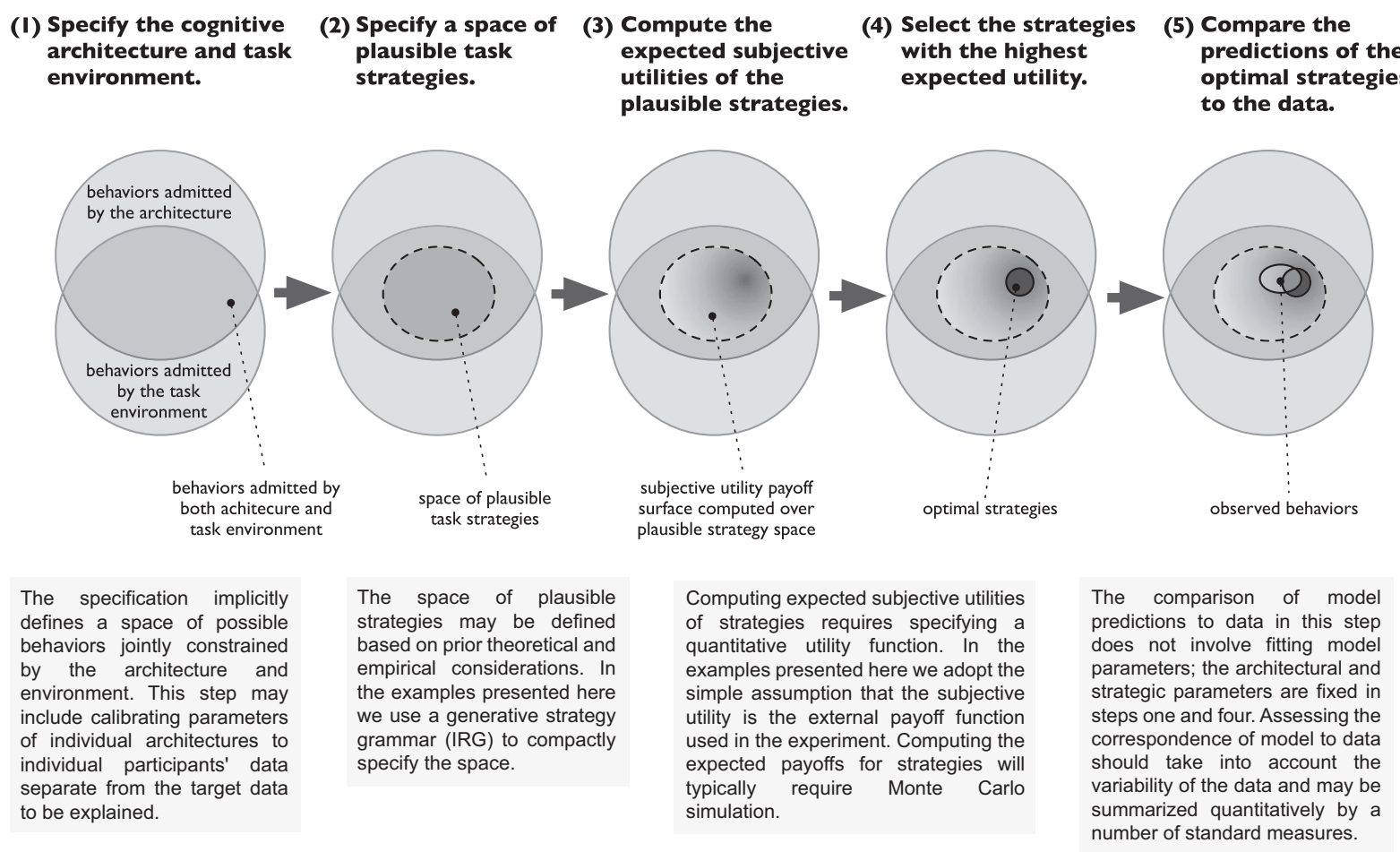

Figure 4. How the five steps of cognitively bounded rational analysis focus the space of behaviors. Step 1 defines architecture and environment. Steps 2-4 narrow that space by first determining the plausible strategies and then determining the subset of best strategies. Only Step 5 involves comparison to data. 


\section{Specify a Theory of the Architecture and Environment and Fix All Architectural Parameters That Can Be Calibrated From the Data}

Step 1 demands a computational or formal specification of an architecture (e.g., ACT-R or EPIC). In the analyses presented in this article we used a specification language called Information Requirements Grammar (Howes et al., 2004, 2005, 2007; Vera et al., 2004). Information Requirements Grammar foregrounds theory-relevant aspects of the specification (e.g., temporal aspects of cognition) and, unlike Lisp or $\mathrm{C}++$, backgrounds theoryirrelevant detail. Step 1 also demands a specification of the environment, minimally including the temporal properties of the stimuli. Lastly, the step demands that architectural parameters are calibrated to some dimensions of the data. Below we describe our methods for calibration and distinguish it from data fitting.

\section{Define and Systematically Generate the Space of Plausible Strategies}

Step 2 demands that all plausible strategies are specified. One possibility would be to use an architecture such as ACT-R or EPIC to code each strategy. However, in the analyses presented below we have again used Information Requirements Grammar, which was specifically designed to support the compact representation of large strategy spaces through hierarchical abstractions.

\section{Determine the Expected Payoff Achieved by Each Model (Strategy + Architecture) With Respect to an Explicit Utility Function}

A formal method in which the consequences of constraints for behavior are derived in some closed-form way may be possible in some cases, but the complex and stochastic nature of theories of the cognitive architecture will usually demand Monte Carlo simulation. The value of the payoff achieved by each model needs to be ascertained to a level of confidence that allows discrimination of the best strategies from the rest.

\section{Select the Subset of Strategies With the Highest Payoff}

Steps 3 and 4 together demand that the specification language supports the expression of a theory of the utility function. The language should not commit to a particular theory; rather it should be possible to express commitments to a particular theory. A recent examples of a hypothesis concerning a utility function is the soft constraints hypothesis of Gray, Sims, Fu, and Schoelles (2006), which states that people seek to minimize time (rather than, e.g., working memory load) in stretches of behavior lasting in the $0.33-\mathrm{s}$ to 3 -s range.

\section{Contrast the Prediction Interval Inferred From the Highest Payoff Strategies With the Asymptotic Human Performance}

Steps 3, 4, and 5 together demand that there is some principled basis for ascribing a specific utility function to the humans performing the task - that is, there must be a theory of the subjective utility function. The most straightforward way of addressing this issue in many cognitive experimental situations is to adopt the practice of providing to participants an unambiguous quantification of the rewards and costs of various behaviors in the task (Sperling \& Dosher, 1986). This approach is exemplified by Schumacher et al. (2001), who used a point scheme that quantified the relative benefits of going fast or avoiding errors and provided participants with incremental feedback throughout the task. Schumacher et al. (2001) further motivated their participants to maximize by giving a cash bonus in direct proportion to the earned points. In such situations, the simplest assumption is that the subjective utility function is identical to the explicit point scheme used in the experiment. We present strong evidence below that this is the case in the four Schumacher experiments.

Step 5 also demands a systematic means of calculating a prediction interval given both within- and between-participants variance (Roberts \& Pashler, 2000).

Finally, and most significantly, it is in Step 5 that the gaps between observed and boundedly optimal performance are revealed. Thus, implicit in Step 5 is the requirement to address these gaps with further refinements to theories of the architecture, environment, strategies, or utility function.

\section{Ordered Response Theory (ORT)}

Using the framework proposed in the previous section, we developed and tested a new theory of the skilled generation of ordered responses, which we applied to four sets of PRP tasks. Unlike existing theories, the new theory offers quantitative predictions and explanations for the effect of SOA on Task 2 duration. It furthermore provides these predictions and explanations at the level of individual participant performance and as such is the first detailed account of individual variability in PRP performance.

The new theory is simply called $O R T$. The theory is that the coordination of ordered responses is a strategic response to a utility function that precisely trades speed for accuracy, given architectural constraints that include variability in individual response times and possibly other response preparation constraints.

The particular models that we focus on here all include internal system noise as the key constraint, but we also test models with varying auxiliary assumptions. When applied to PRP performance, these theories predict that people separate the Task 2 response by just enough time to optimize subjective expected utility given the distributions of RT1 and RT2 and the response preparation limitations. Given a defined utility function, a quantitative prediction of the deferment duration can be made by optimizing the function given the constraints.

The ORT is inspired by Meyer and Kieras's (1997a) strategic response deferment account and shares the assumption that participants achieve response ordering by postponing the Task 2 response. However, it differs in two respects. First, in the models reported by Meyer and Kieras (1997a) the duration that Task 2 was postponed was fit to the data. Therefore, although Meyer and Kieras (1997a) emphasized the potential role of this strategic interval in avoiding reversals, their approach did not provide a basis for predicting its duration.

Second, in Meyer and Kieras's (1997a) strategic response deferment theory, it was assumed, as the name suggests, that some part of Task 2 was deferred until after some part of Task 1 was completed. The Task 2 response was not initiated until after a 
control signal from Task 1 had been received and the strategic interval had been observed. In contrast, ORT does not make a commitment to whether Task 2 is deferred until after some part of Task 1 or to whether the Task 2 response is merely delayed. If the response is delayed, then a strategic interval is observed, but the start of this interval is not contingent on a control signal from the partial completion of Task 1 .

\section{A Test of ORT}

In this section of the article, we develop and test the ORT using a CBR analysis. The method involves the five steps specified above. The work done for each step is explained in the next five sections.

\section{A Theory of the Architecture (Step 1)}

We are particularly interested in aspects of the architecture that may support strategies for PRP performance and, more generally, for accomplishing response ordering. There are various auxiliary assumptions that need to be considered. Response ordering strategies imply (a) an architecture with a timing capability (the duration by which to defer or delay) and (b) the availability of control signals arising from partial completion of a stimulus-response. We also expect response ordering to be sensitive to whether or not there is a bottleneck constraint on central cognition, and for the moment we commit to the EPIC assumption that there is no central bottleneck. We later explore the implications of this commitment.

\section{Structure of the Architecture}

We take EPIC (Meyer \& Kieras, 1997a, 1997b) as our point of departure for specifying the architecture. The key assumptions we adopt from EPIC concern the processing stages required for accomplishing a PRP task, including their relative durations. We describe below how we used these assumptions to build a constrained parametric architecture that is capable of accommodating individual differences in stage durations while maintaining key relationships among the durations. We refer to this specific set of assumptions as EPIC-V because it incorporates assumptions about the variance of process durations that differ from EPIC assumptions. Where EPIC used no variance in perceptual and cognitive processes and average variance in motor processes, EPIC-V uses calibrated variance in perceptual, cognitive, and motor-processing stages, as described in detail below. ${ }^{4}$

The initial version of EPIC-V that we explore here is defined by the following assumptions. The assumptions provide the default quantitative parameters associated with process durations; in the section below on calibration, we describe precisely how a subset of these parameters are adjusted in a constrained way for individual participants.

1. The default auditory identification duration was set to $209 \mathrm{~ms}$, and the default visual identification duration was set to $258 \mathrm{~ms}$ (the means of two sets of five values used in Meyer and Kieras, 1997b).

2. There was no response selection bottleneck. (Later in this article we test the sensitivity of the predictions to this assumption by running the models with a response selection bottleneck.)

3. There was no postcontrol processing bottleneck if responses were in different modalities (e.g., in E4 participants made a vocal and a manual response). If responses were in the same modality (E1-E3), then process interference, or jamming, occurred (Klapp, Nelson, \& Jagacinski, 1998; Meyer \& Kieras, 1997a) if Task 2 motor response processes started before Task 1 motor processing had completed. In the simulations we adopt the simple assumption that process interference results in an error trial. As with the selection bottleneck assumption, we also test the sensitivity of the predictions to this assumption by running the models without interference.

4. We assumed that the selection cycle took a default of 50 $\mathrm{ms}$ and that for binary choice tasks 1.12 selection cycles were required (this is the average of the four values used in Meyer and Kieras, 1997b). For the easy numerosity task, we assumed 1.00 selection cycle, and for the hard numerosity task, we assumed 5.00 selection cycles. These values were the same as those used by Meyer and Kieras (1997b) to model Hawkins, Rodriquez, and Reicher (1979), who reported an experiment with eight mappings in the hard condition (similar to Schumacher et al., 1999). For the easy stimulus-response compatibility task, we used 1.00 selection cycle, and for the hard stimulus-response compatibility task, we used 2.23 cycles. These were the values used by Meyer and Kieras (1997b) to model McCann and Johnston's (1992) Experiments 1 and 2. Again, these researchers used similar compatibility tasks to those used by Schumacher et al. (1999).

5. The default working memory gating time was set to 25 ms. In EPIC, this corresponds to the mean time that a result placed in working memory must wait to be processed by cognition given the 50 -ms production firing cycle (Meyer \& Kieras, 1997a).

6. The motor-processing stage corresponds to Meyer and Kieras's (1997a) motor-processing stage. For E1, E2 and E3, in which Task 1 and Task 2 share response modalities, we set the mean default duration of the motor preparation process for the first task to $150 \mathrm{~ms}$ and the mean duration of the motor preparation process for the second task to $100 \mathrm{~ms}$. The value is shorter for the second task because the motor system is assumed to be ready (Byrne \& Anderson, 2001; Meyer \& Kieras, 1997a, p. 17). For E4, in which Task 1 and Task 2 have different response modalities, we set the mean default duration of the motor preparation process to $150 \mathrm{~ms}$ for both tasks.

\footnotetext{
${ }^{4}$ Variability plays an important role in the modeling of Anderson, Taatgen, and Byrne (2005)
} 
7. The motor initialization process was assumed to have a default mean duration of $50 \mathrm{~ms}$ (the production firing cycle time in EPIC).

8. Manual transduction was set to a default duration of 20 ms (an average of three values used by Meyer and Kieras, 1997b), and vocal transduction was set to a default of $80 \mathrm{~ms}$ (average of three values used in Meyer and Kieras, 1997b).

9. The unlock process switches from deferred to immediate mode and has a mean duration of $100 \mathrm{~ms}$ (see Table 1 in Meyer \& Kieras, 1997a).

10. All information flows between processes were mediated by buffers of unbounded duration.

11. All process durations were sampled from gamma distributions (Van Zandt \& Ratcliff, 1995), the shape and scale of which were calibrated from the data (more details are provided in the following calibration section below).

\section{Calibration of the Architectural Parameters}

The idea behind calibration is straightforward: distinguish between a set of outcome variables whose patterns represent the phenomena to be explained and a set of calibration variables whose patterns will be used to determine architectural parameter settings that will remain fixed for the prediction of the outcome variables. The theory is thereby subjected to greater test because the quantitative theoretical degrees of freedom cannot be used to fit the target pattern of data in the outcome variables. Put another way, the theory predicts a necessary relationship between the outcome and calibration variables, and this relationship is put to the test. In contrast, traditional model fitting adjusts the architectural parameters in order to best match the pattern in the outcome variables, but as argued earlier this can lead to the interpretation problems identified by Roberts and Pashler (2000).

Calibration is not a new idea. It was adopted to some extent, for example, in Byrne and Anderson (2001), it is consistent with the Roberts and Pashler (2000) recommendations, and it is closely related to methods in machine learning and allied fields that distinguish in some way between training and test data sets. What we are suggesting here is that calibration be made a systematic part of modeling done in concert with CBR analysis, so that both architectural and strategic degrees of freedom are simultaneously constrained in a principled fashion.

The central claim of the ORT is that people adapt to their own idiosyncratic process means and noise signatures, so we created individual instantiations of the architecture that were calibrated separately for each participant. We were interested in predicting the shape of the PRP curve, so the outcome variables are Task 2 performance at 50-, 150-, 250-, and 500-ms SOA, and we calibrate to $1,000-\mathrm{ms} \mathrm{SOA}$ only. None of the models' parameters were fit to performance at 50-, 150-, 250-, or 500-ms SOA.

The calibration was based on data from the last session of performance (in Schumacher et al., 1999, there were two sessions for E1 and three sessions for E2 to E4). Each block consisted of 40 trials irrespective of experiment. Some experiments had more sessions and more blocks than others; there was therefore some variation in the level of expertise achieved by participants.

Figure 5 shows a sample of the RT distributions of one particular participant. We calibrated to mean RT and variance at 1,000-ms SOA, using the following method.

$R T$ calibration. For each experiment, we used the calibration to generate model durations for 9 processes (Task 1 perceive, select, motorprep, and motorinit; Task 2 perceive, easy select, hard select, motorprep, and motorinit) given EPIC default durations (see above) and observed RTs at 1,000 ms. We used a mixed effects fitting procedure based on maximum-likelihood estimation to generate estimates of the process durations for each participant (Specifically, we used the nlme function in R; Lindstrom \& Bates, 1990; Pinheiro, Bates, DebRoy, Sarkar, \& the R Core team, 2009). Mixed effects model fitting was chosen because it provides better and more constrained estimates of individually varying parameters (see, e.g., Gelman \& Hill, 2007).

The calibration was subject to the following theoretical constraints: (a) The ratio of the calibrated Task 1 motor process duration to the calibrated Task 2 motor process duration was set to the corresponding default motor process ratio, and (b) the ratio of the calibrated select durations to the default motor process durations was held constant. These ratios are critical to the predicted SOA effect. Ratio (a) is important because the relative duration of the motor processes is central to determining the duration by which Task 2 must be deferred in order to ensure that no response reversal occurs. Ratio (b) is important because the duration of the select processes is central to determining the effect, or otherwise, of a selection bottleneck.

Variance-to-mean ratio (VMR). While ACT-R and EPIC include hypotheses concerning mean process duration, they are less specific about the variance of these durations. In EPIC, process variance is uniform and limited to the motor system. In ACT-R, there is also variance in the duration required for retrieval. Variance in ACT-R's production cycle could be set with a parameter, though the values of this parameter have not been systematically explored in ACT-R models. For this reason, and unlike for duration, there was not a set of default variances for us to use as a starting point for calibration. Instead, we chose to distribute the observed variance of the RT proportionately across the hypothetical constituent processes; that is, we assumed a constant VMR (similar to a constant coefficient of variation, a quantity expressed in terms of standard deviation used by Schweickert, Fisher, \& Proctor, 2003) across all cognitive, perceptual, and motor processes. This assumption may need to be relaxed in future modeling, but it represents the simplest and most constrained starting point.

VMR gives a measure of variance per unit process duration. We assumed that there was a positive linear relationship between duration and its variance and therefore calculated the VMR for each participant's Task 1, Task 2 easy, and Task 2 hard RT. In addition, we calculated a mean VMR for each participant (across Task 1 and the two conditions of Task 2) as well as a mean Task 2 VMR (averaged over easy and hard).

For each participant, the variance of each process in the model was determined by multiplying the appropriate VMR by the scaled process duration. More specifically, (a) the variances of Task 1 perceive, response selection, and motor prepare processes were calculated by multiplying the respective mean duration by the 
E1 1 easy 50

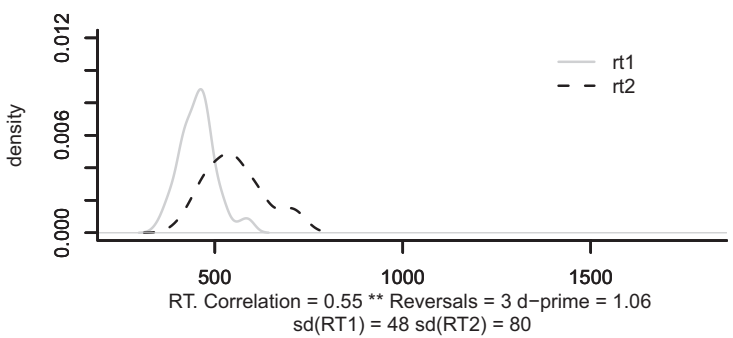

E1 1 easy 150

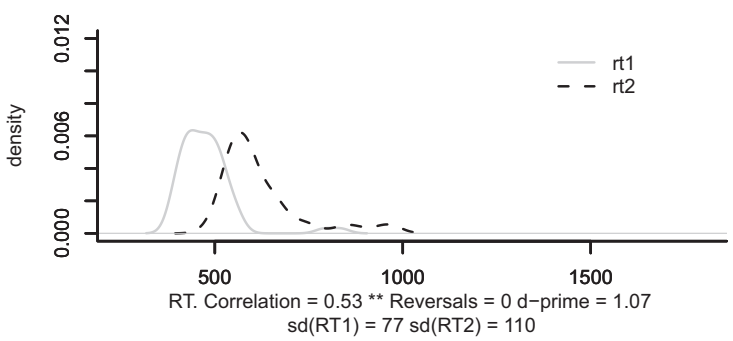

E1 1 easy 250

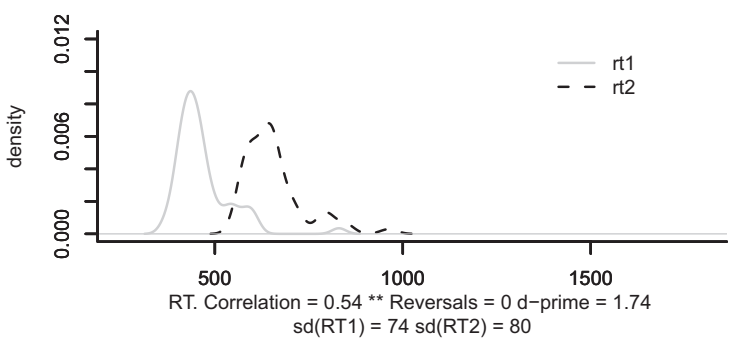

E1 1 easy 500

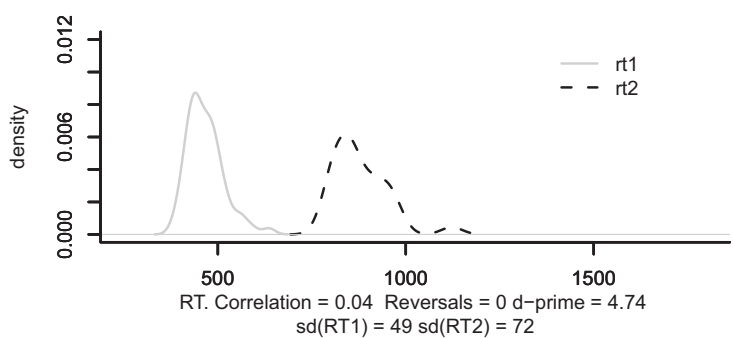

E1 1 easy 1000

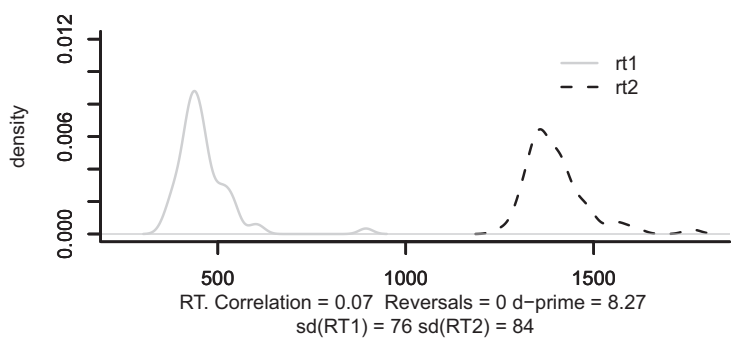

E1 1 hard 50

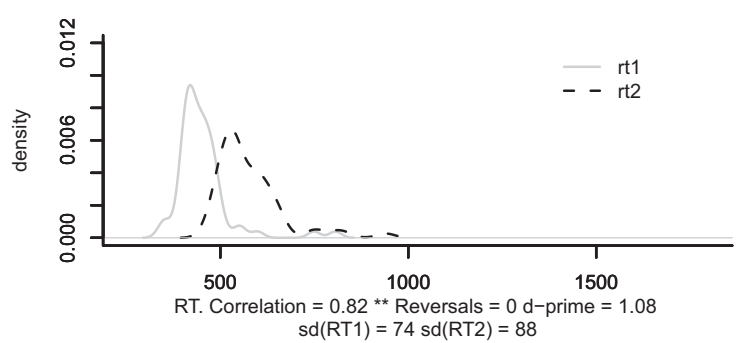

E1 1 hard 150



E1 1 hard 250

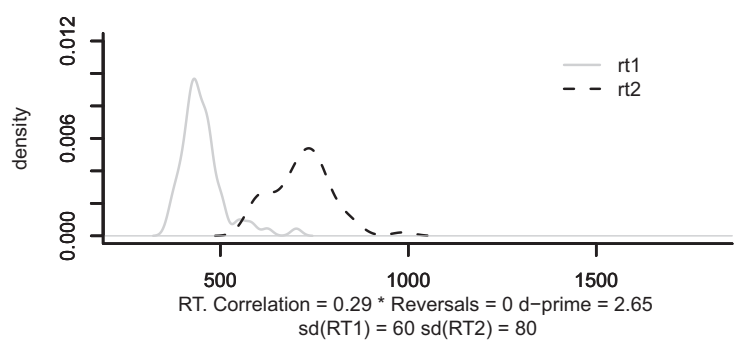

E1 1 hard 500

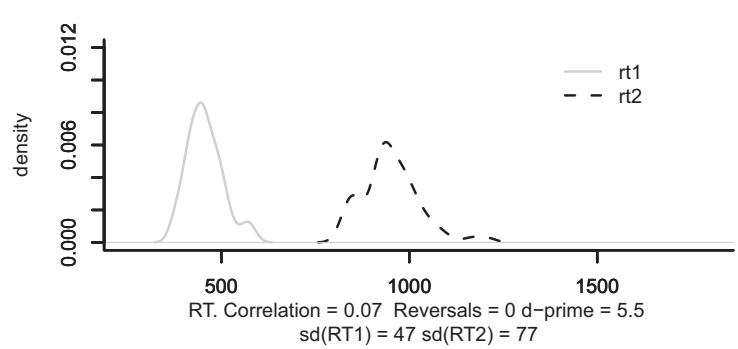

E1 1 hard 1000

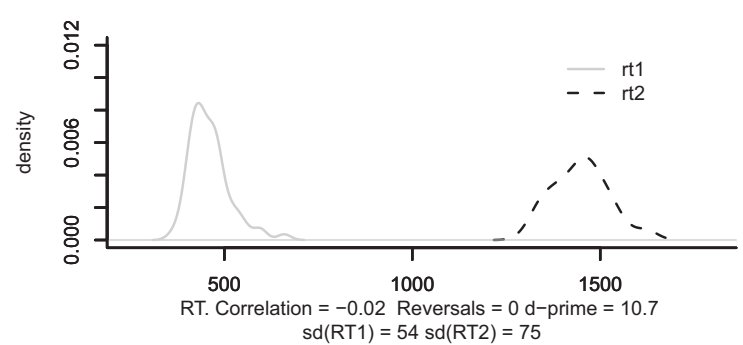

Figure 5. Density plots of Task 1 response time (RT1) and Task 2 response time (RT2) from Experiment 1 (E1) Participant 1 easy and hard conditions across all five levels of stimulus onset asynchrony. sd $=$ standard deviation. 
VMR for Task 1; (b) the variances of Task 2 perceive, response selection, and motor prepare processes were calculated by multiplying the respective mean duration by the VMR for Task 2; (c) the variances for motorinit processes, which are shared between Task 1 and Task 2, were calculated by multiplying the mean duration by the VMR for both tasks; and (d) the variances of executive processes (defer and unlock) were calculated by multiplying the mean duration by the VMR for both tasks.

Skew and kurtosis parameters. Having calculated a mean and variance for each process we then calculated the skew and kurtosis of the gamma distribution, from which instances of the duration would be sampled, following assumptions in Weisstein (2008).

\section{The Space of Plausible Strategies (Step 2)}

We assumed that there were two dimensions of strategic variation. The first was the positioning of the Task 2 perception, selection, and motor processes relative to the deferred processing stage. We assumed that either (a) the whole of Task 2 was deferred (Task 2 perception, selection, and motor processes were deferred), (b) only response selection and motor processing were deferred, or (c) only motor processing was deferred. The second dimension of variation was the duration of the deferred processing stage. The defer process duration ranged between $2 \mathrm{~ms}$ and $825 \mathrm{~ms}$, at 25-ms intervals. The $25-\mathrm{ms}$ grain size is somewhat arbitrary, but our subsequent results will show that a finer grain size is unlikely to produce significantly better predictions.

We assumed that the participant's subjective utility function was determined precisely by the experimental payoff regime reported by Schumacher et al. (1999). On errorless trials, participants received points worth $[100-(\mathrm{RT} 1 / 10)]+[100-(\mathrm{RT} 2 / 10)]$. In this way, the payoff rewards speed equally for Task 1 and Task 2 . Response reversals or other types of error resulted in the deduction of 100 points; in this way, the payoff rewards accuracy. The payoff thus provides participants with a precise quantitative specification of the relative rewards of speed and accuracy. Participants received an additional 1,000 points if the mean of RT1 at $50 \mathrm{~ms}$ for a block was within $75 \mathrm{~ms}$ of the mean of RT1 at 1,000 ms.

\section{Determining Expected Payoffs (Step 3)}

We determined the expected payoff for each of the strategies for each participant using 5,000 trials of Monte Carlo simulation (1,000 per level of SOA) for each condition (easy-hard) of each experiment, separately for each individual participant. There were 8 participants in E1, 10 in E2, 8 in E3, and 6 in E4. The expected payoff of each strategy was the mean of the expected payoff at each level of SOA; each SOA appeared equally often in the experiments, so a weighted mean was not required.

\section{Selecting the Highest Payoff Strategies (Step 4)}

We defined the best strategy for a given individual in an experimental condition as the strategy that achieved the highest expected payoff according to Schumacher et al.'s (1999) payoff regime.

\section{Comparing the Predictions to the Human Data (Step 5)}

In many of the analyses presented below, we focus specifically on the fourth experiment, E4. The design of E4 provides the best opportunity to test the variance-bounded assumption because there were no perceptual-motor conflicts between Task 1 and Task 2. If some evidence in favor of the theory can be found by examining the results of E4, then E1, E2, and E3 allow us to further test the auxiliary architectural assumptions, including the motor jamming assumption. Each of these three experiments exhibits one or other modality conflict, and given that our models use the EPIC jamming assumption, the experiments provide an opportunity for testing this assumption.

In what follows, we first test the theory with all of the architectural assumptions detailed above and then subsequently attempt to isolate the effects of the selection bottleneck and jamming assumptions. We initially focus on how well the theory predicts the PRP curve (RT vs. SOA), first for individuals, then in aggregate. The aggregate predictions are generated from the individual predictions but serve to make clear the source of the shape of the aggregate PRP curves presented in Schumacher et al. (1999). Finally, we examine in more detail the nature of the payoff curves for the individual subjects in E4.

\section{Task 1 RT as a Function of SOA}

Recall that according to Schumacher et al.'s (1999) payoff regime, participants were awarded an extra 1,000 points if their mean RT1 at $50-\mathrm{ms} \mathrm{SOA}$ was within $75 \mathrm{~ms}$ of RT1 at $1,000-\mathrm{ms}$ SOA. All participants in E2, E3, and E4 achieved this criterion correspondence (flat RT1). Two participants in E1 failed to achieve flat RT1. These two participants were excluded from further analysis because the models do not make predictions about circumstances in which this criterion is not met. (As a consequence, the mean RTs for E1 reported below do not correspond to those reported by Schumacher et al., 1999.)

\section{RT2 as a Function of SOA}

Roberts and Pashler (2000) advocated the identification of an a priori plausible range of possible outcomes against which to compare a model's prediction interval. In a Bayesian sense, making explicit such a plausible range helps to reveal the prior probability of observing some set of outcomes; the tighter the prediction interval relative to the plausible range, the stronger the theoretical prediction and the greater the justified increase in belief in the theory if the data correspond to the prediction. In the remaining analyses, we define plausible outcomes as those in the space between, and including, perfect time-sharing and serial processing. As one will see, this range receives some empirical justification because some observed individual participant's data lie at each boundary. We first present a detailed analysis of the individual performance in E4 and then give an overview of individual performance on E1-E3.

The models for each participant in the hard condition of E4 are compared to the RT data in Figure 6. It is important to keep in mind that the model predictions are derived from the strategy that generated the highest payoff using a Monte Carlo simulation and given the calibrated architectures. The predictions are not the result of effort to maximize $R^{2}$ or any other measure of model-data match.

In Figure 6 there are six panels, one for each participant in E4. Each panel shows a plot representing the participant's data with 
E4 hard subject 1

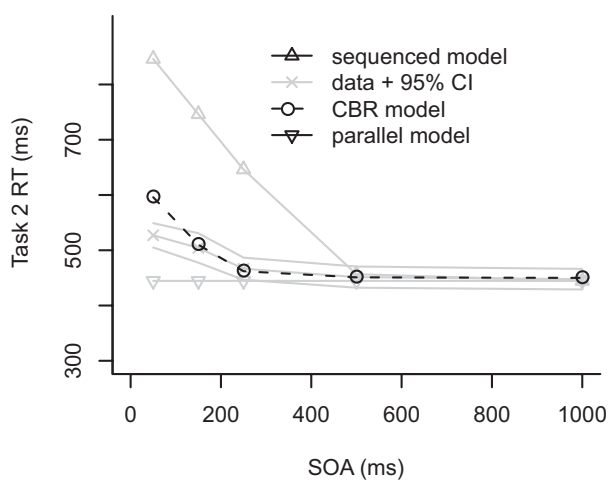

E4 hard subject 3

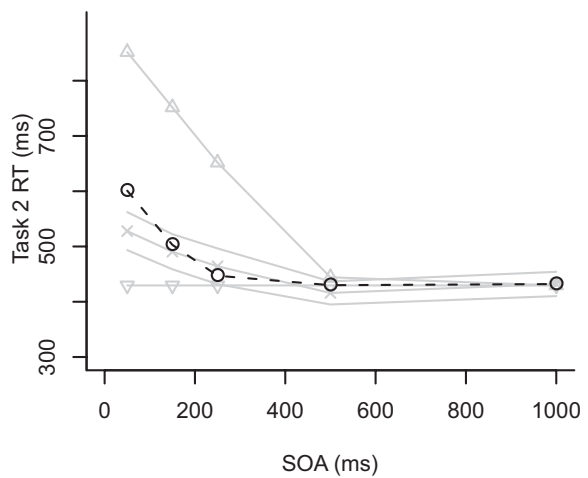

E4 hard subject 5

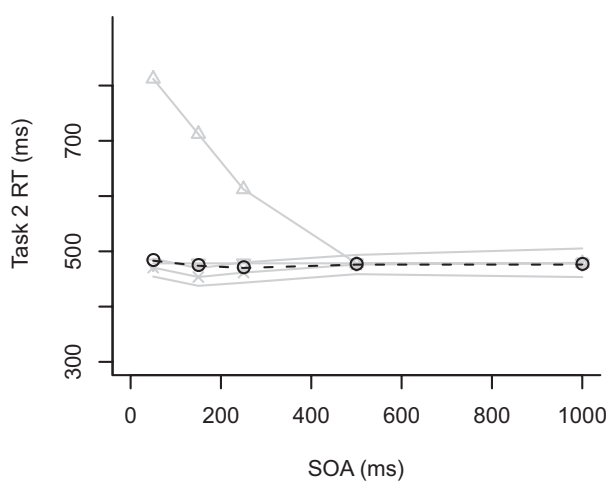

E4 hard subject 2

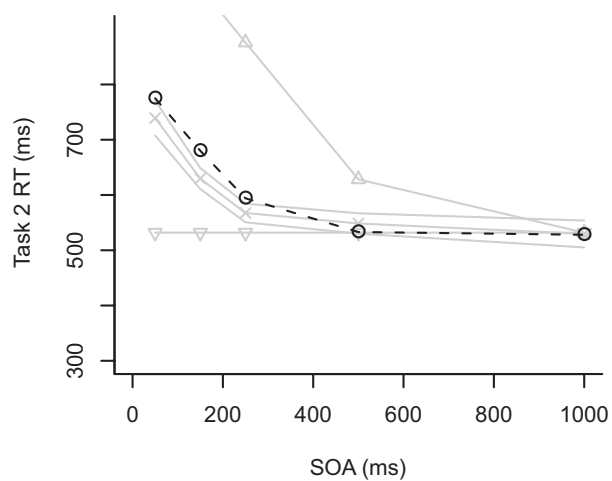

E4 hard subject 4

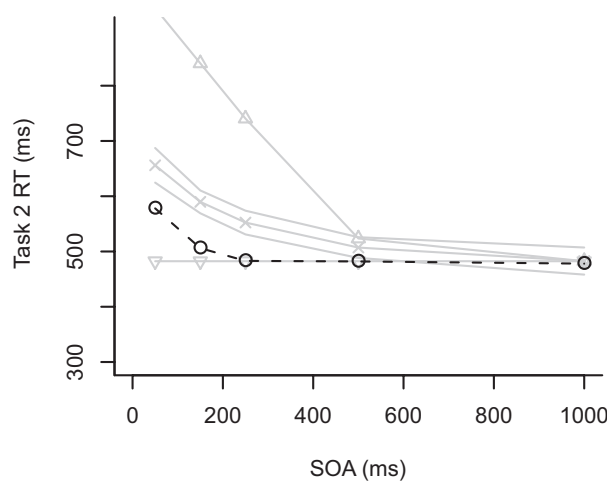

E4 hard subject 6

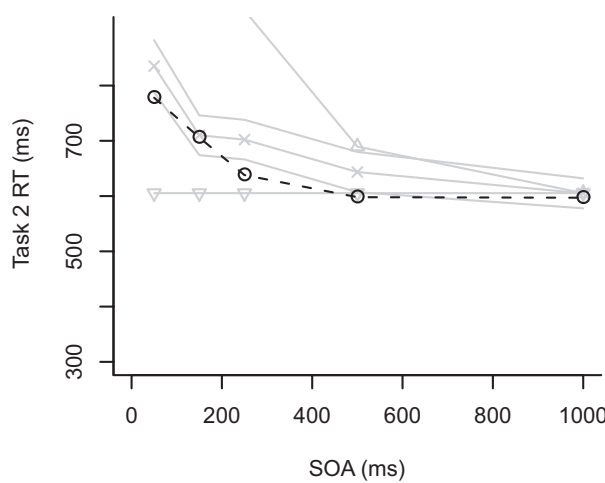

Figure 6. Cognitively bounded rational (CBR) ordered response theory predictions and individual human data for Task 2 response time (RT) as a function of stimulus onset asynchrony (SOA) and Task 2 difficulty (hard condition only). The data are reanalyzed from Schumacher et al.'s (1999) Experiment (E) 4. 95\% CIs (confidence intervals) are shown for data only. The 95\% confidence intervals for the model were smaller than for the data and are not shown for clarity.

95\% confidence intervals based on the variability within each condition and a plot representing the prediction of the cognitively bounded model. In addition, two other models are plotted. These represent the span of the strategy space from highly parallel to highly serial processing, and therefore give an indication of the plausible strategy space.
Figure 6 shows that the data from 17 of 24 sub-1,000-ms SOA conditions for the 6 participants lie within the prediction interval of the cognitively bounded model for E4 hard. The worst exception was Participant 4 . The figure also shows that the correspondence between the data and the model was not inevitable: The range of plausible outcomes is represented by the space between the par- 
allel model and the serial model. The prediction interval for the theory is much tighter than this plausible range because all strategies other than the represented prediction were eliminated using the CBR analysis.

Further, there is some evidence from Participants 1, 2, 3, and 5 that the model predictions capture the shape of the PRP curve and its variations from individual to individual. This variation is considerable; there is no single canonical PRP curve. We will see below that, although not all RTs match the predictions, they are nevertheless on the predicted payoff curve and still quite close to optimal.

The quality of the prediction at 50-ms SOA is not simply a function of the fit of the model to RT2 at long SOA. For example, a fast RT2 at 1,000 ms does not mean a fast RT2 at short SOA. This fact is most clearly illustrated by the flat relationship between RT2 and SOA in Participant 5. The effect of SOA is negligible for Participant 5 compared to its effect in Participant 3, who actually had faster RTs at longer SOAs. In general, the variation in RT2 at $1,000-\mathrm{ms} \mathrm{SOA}$ is not sufficient to explain its variation at $50-\mathrm{ms}$ SOA.

The performance of E4's Participant 5 is unusual and warrants further attention. For this participant, we examined the contribution of variance and RT1 and RT2 duration to the effect of SOA. We found that the variance was the lowest of any of the participants in E4. We also found that this participant had the fastest RT1 relative to RT2. Both of these factors will tend to reduce the impact of Task 1 on Task 2 at short SOA. Low variance and a faster RT1 both reduce the probability of a response reversal and therefore reduce the need for strategic delay.

Recall that the prediction of performance at 50-ms SOA is the prediction of the strategy with the highest utility given the architectural and environmental assumptions. Of the $3 \times 33$ strategies (Perceive, Select, or Motor Control Point $\times$ Durations of Defer) that were considered as candidate best strategies, the best strategies for each of the participants in the easy condition corresponded to defer durations of $50 \mathrm{~ms}, 75 \mathrm{~ms}, 50 \mathrm{~ms}, 25 \mathrm{~ms}, 25 \mathrm{~ms}$, and $100 \mathrm{~ms}$, respectively. Thus, the same strategy was not best for all participants.

\section{Individual Predictions for E1-E4}

So as to gain a more quantitative description of the quality of the predictions for all 30 of the participants in E1-E4, we calculated values of $R^{2}$ and RMSE for each individual. ${ }^{5}$ The values are presented in Table 1. Each value is for 10 data points (5 levels of SOA for each of 2 levels of task difficulty). Table 1 shows the $R^{2}$ and RMSE values for three different models of the individual participants in all four experiments. Focus for now on the values for the no bottleneck-jamming model. Many, though not all, of the $R^{2}$ values are high (24 out of the 30 are over 0.80 ), suggesting that the models offer reasonable explanations for the shape of the two SOA curves for each participant (up to a linear transformation).

Two predictions, those for Participant 7 in E2 and Participant 1 in E3, resulted in particularly low values of $R^{2}$, suggesting that the model did not provide a good account of performance in these cases. Examination of the individual RT versus SOA plots for these participants revealed that both were slower at 50-ms SOA than the sum of Task 1 and RT2 at 1,000-ms SOA (i.e., it would have been faster for them simply to sequence their two responses).
Thus, they are outside our predefined plausible range. On mean the models account for $89 \%, 84 \%, 77 \%$, and $85 \%$ of each individual's variance due to SOA, in each of the four experiments, respectively.

We also calculated the proportion of the between-participants variance that was explained by the model for each experiment. The $R^{2}$ values were $0.02,0.27,0.08$, and 0.66 , respectively, indicating that the model accounted well for the between-participants variance in E4 but not at all for the variance in E1, E2, and E3. One reason that the $\mathrm{E} 4$ prediction is good and the others are not may be that this experiment exhibited the largest between-participants variance. There was relatively little between-participants variance to be explained in E1-E3.

\section{Predicting Across-Participants Means for RT2}

Although the previous analysis shows how the cognitively bounded model can predict individual performance, we can also make an assessment of how well it predicts aggregate performance. This kind of aggregate analysis is useful because it is more directly comparable to existing accounts that do not model individual variation and because it may reveal systematic biases in the departure of the models from the data.

Figure 7 shows mean RT as a function of SOA for Task 2 across all four experiments. There are eight panels, including an easy and a hard plot for each experiment. The data are shown with the between-participants 95\% confidence interval, and the model's aggregate prediction is the mean of the predictions for each participant-that is, the mean of the best strategies for each participant (where, again, best is defined in terms of payoff, not fit to data). The data are within the prediction interval for five of the eight conditions across the four experiments. Importantly, there is a good correspondence between data and model for E4, which provides the critical, modality-conflict free test of the variance assumption in the cognitively bounded account. Although the confidence band for E4 is relatively wide, the individual analyses above indicate why: The variance is due to the inclusion of participants such as 2 and 5, each with strikingly different data patterns. But this variance is systematic and accounted for by the theory.

The conditions for which the data are not within the prediction interval of the theory are the hard conditions of experiments with a shared response modality (E1, E2, and E3). These cases may point to the possibility that the EPIC-V architecture is underconstrained.

\section{Payoff as a Function of Interresponse Interval}

The CBR analysis provides an alternative way of viewing the data that gives further insight into the PRP phenomena. It is possible to create individual payoff curves that plot expected payoff against some variation in strategy, rather than focus exclusively on the optimal prediction. Plotting such payoff curves, along with the observed data, is important for three reasons: (a) It allows one to see the correspondence between the optimal prediction and the actual data in the context of the theoretical payoff space; (b) it

\footnotetext{
${ }^{5}$ We use RMSE rather than root-mean-square deviation because there is no variation in number of parameters between the reported models (see Pitt, Myung, \& Zhang, 2002, p. 475).
} 
Table 1

$R^{2}$ and Root-Mean-Square Error (RMSE) Between Model and Observed Task 2 Response Time for Each Participant in All Four Experiments Across All Levels of Stimulus Onset Asynchrony (SOA) for Each of Three Models: Bottleneck-Jamming, No BottleneckJamming, and No Bottleneck-No Jamming

\begin{tabular}{|c|c|c|c|c|c|c|}
\hline \multirow[b]{2}{*}{ Participant } & \multicolumn{3}{|c|}{$R^{2}$} & \multicolumn{3}{|c|}{ RMSE } \\
\hline & Bottleneck-jamming & $\begin{array}{c}\text { No } \\
\text { bottleneck-jamming }\end{array}$ & $\begin{array}{c}\text { No } \\
\text { bottleneck-no jamming }\end{array}$ & Bottleneck-jamming & $\begin{array}{c}\text { No } \\
\text { bottleneck-jamming }\end{array}$ & $\begin{array}{c}\text { No } \\
\text { bottleneck-no jamming }\end{array}$ \\
\hline \multicolumn{7}{|c|}{ Experiment 1} \\
\hline 1 & 0.96 & 0.94 & 0.95 & 24 & 34 & 36 \\
\hline 2 & 0.92 & 0.83 & 0.74 & 59 & 78 & 99 \\
\hline 3 & 0.89 & 0.89 & 0.92 & 30 & 30 & 33 \\
\hline 4 & 0.92 & 0.83 & 0.81 & 96 & 118 & 124 \\
\hline 5 & 0.88 & 0.90 & 0.91 & 40 & 34 & 29 \\
\hline 6 & 0.96 & 0.93 & 0.93 & 56 & 78 & 79 \\
\hline$M$ & 0.92 & 0.89 & 0.88 & 51 & 62 & 67 \\
\hline \multicolumn{7}{|c|}{ Experiment 2} \\
\hline 1 & 0.99 & 0.88 & 0.86 & 11 & 32 & 46 \\
\hline 2 & 0.92 & 0.84 & 0.80 & 32 & 42 & 50 \\
\hline 3 & 0.95 & 0.95 & 0.97 & 19 & 18 & 27 \\
\hline 4 & 0.94 & 0.88 & 0.68 & 16 & 25 & 40 \\
\hline 5 & 0.93 & 0.85 & 0.71 & 43 & 53 & 65 \\
\hline 6 & 0.90 & 0.93 & 0.84 & 31 & 24 & 28 \\
\hline 7 & 0.37 & 0.40 & 0.26 & 86 & 84 & 92 \\
\hline 8 & 0.87 & 0.79 & 0.65 & 29 & 37 & 44 \\
\hline 9 & 0.97 & 0.97 & 0.77 & 11 & 14 & 36 \\
\hline 10 & 0.95 & 0.95 & 0.91 & 15 & 20 & 27 \\
\hline$M$ & 0.88 & 0.84 & 0.75 & 29 & 35 & 46 \\
\hline \multicolumn{7}{|c|}{ Experiment 3} \\
\hline 1 & 0.39 & 0.25 & 0.17 & 222 & 233 & 241 \\
\hline 2 & 0.85 & 0.86 & 0.63 & 63 & 65 & 86 \\
\hline 3 & 0.79 & 0.65 & 0.58 & 68 & 85 & 94 \\
\hline 4 & 0.99 & 0.98 & 0.94 & 26 & 19 & 67 \\
\hline 5 & 0.89 & 0.89 & 0.83 & 65 & 68 & 84 \\
\hline 6 & 0.85 & 0.83 & 0.85 & 47 & 66 & 64 \\
\hline 7 & 0.94 & 0.89 & 0.82 & 39 & 48 & 62 \\
\hline 8 & 0.88 & 0.77 & 0.92 & 46 & 59 & 47 \\
\hline$M$ & 0.82 & 0.77 & 0.72 & 72 & 80 & 93 \\
\hline \multicolumn{7}{|c|}{ Experiment 4} \\
\hline 1 & 0.90 & 0.87 & 0.87 & 22 & 37 & 37 \\
\hline 2 & 0.99 & 0.98 & 0.98 & 9 & 29 & 29 \\
\hline 3 & 0.88 & 0.84 & 0.84 & 40 & 48 & 48 \\
\hline 4 & 0.93 & 0.89 & 0.89 & 32 & 48 & 48 \\
\hline 5 & 0.67 & 0.59 & 0.59 & 40 & 36 & 36 \\
\hline 6 & 0.94 & 0.92 & 0.92 & 39 & 47 & 47 \\
\hline$M$ & 0.89 & 0.85 & 0.85 & 30 & 41 & 41 \\
\hline
\end{tabular}

allows one to see the shape of the payoff curve that, according to the theory, participants are being asked to adapt to; and (c) it allows one to see whether the actual data are on the payoff curve, even when it is not at maximum. This provides some information about whether the participants are in fact navigating the posited payoff space, and from which part of the space they are approaching asymptote.

In the present case, we exploit the fact that the models also predict interresponse interval (IRI), the gap between RT1 and RT2. The IRI is the directly observable variable that is closest in duration to the underlying strategic deferment time. In Figure 8 the payoffs are plotted as a function of IRI at 50-ms SOA for E4 hard participants. We focused in particular on predictions of perfor- mance at 50-ms SOA because, given the calibration of RT2 to $1,000-\mathrm{ms} \mathrm{SOA}$, it is at $50 \mathrm{~ms}$ that we see the strongest test of the theory. The model is somewhat more likely to predict the data at $150 \mathrm{~ms}, 250 \mathrm{~ms}$, and 500-ms SOA (i.e., as the SOA approaches the value used to calibrate the model).

For each experiment, there are two interesting predictions to consider. The first prediction is that participant performance would correspond closely to the model RT at the highest possible payoff. The second prediction is that, even if participant performance is not at the highest value predicted, it would still be on the payoff curve. There are two basic reasons that this prediction could fail to hold. It might be that the architectural theory is wrong. For example, the variance at SOA of $1,000 \mathrm{~ms}$ may not have the 
E1 easy

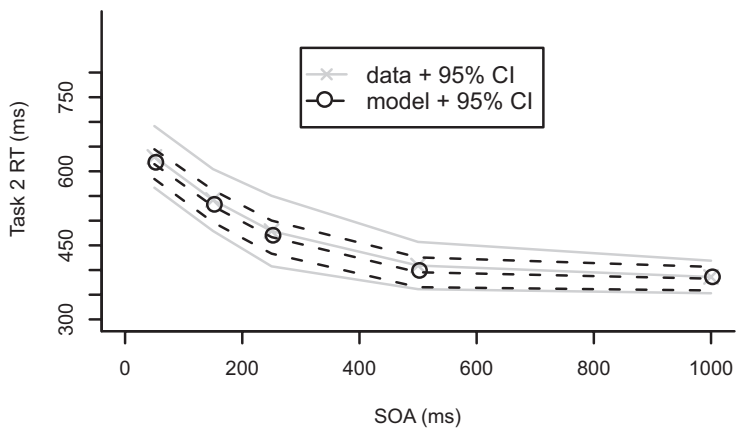

E2 easy

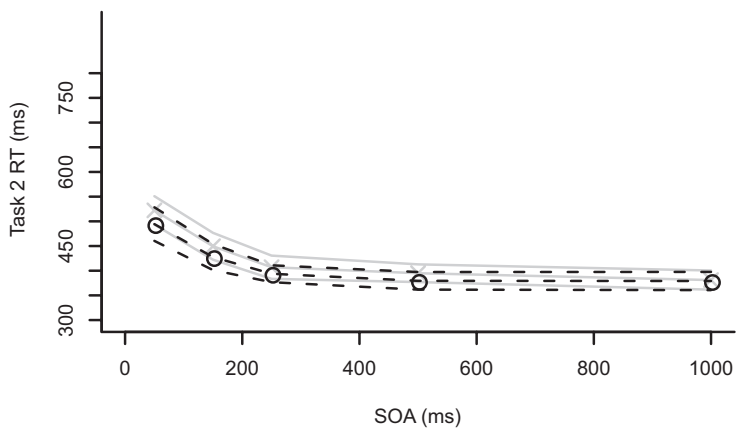

E3 easy

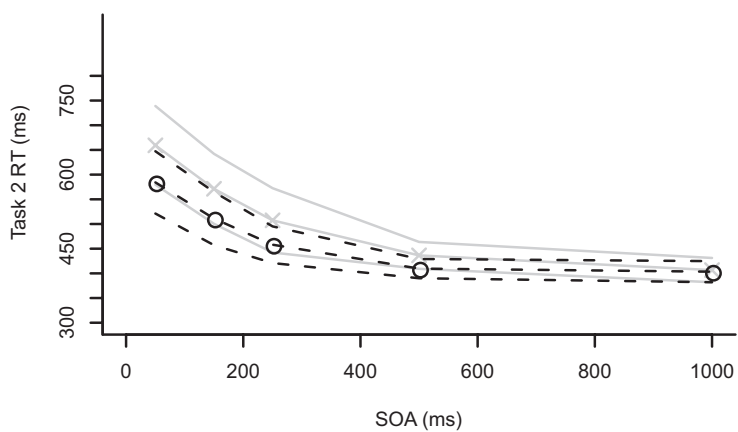

E4 easy



E1 hard

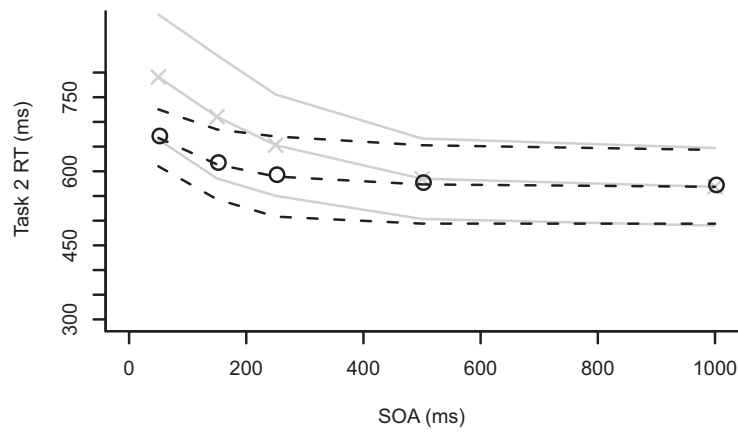

E2 hard

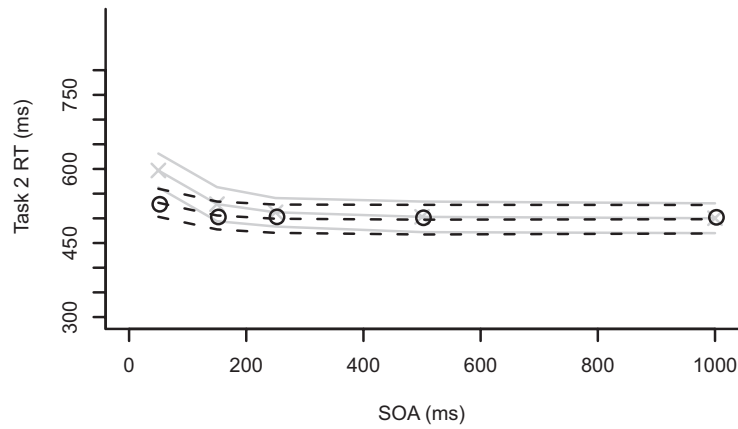

E3 hard



E4 hard

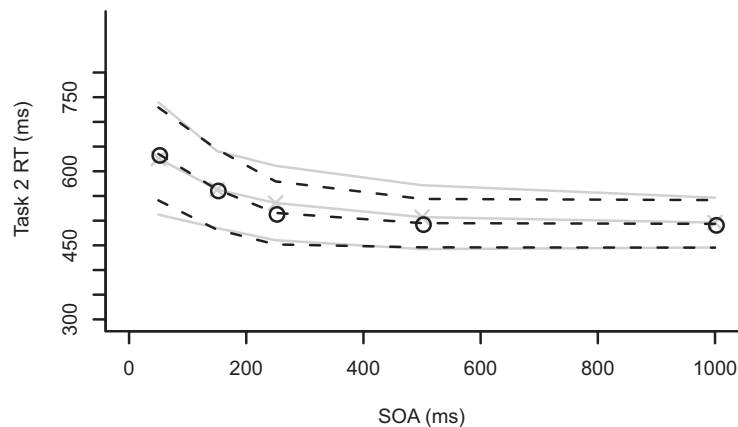

Figure 7. No-bottleneck model predictions and mean human data for Task 2 response time (RT) as a function of stimulus onset asynchrony (SOA) and Task 2 difficulty (easy vs. hard). The data are reanalyzed from Schumacher et al.'s (1999) Experiments (E) 1-4. The 95\% CIs (confidence intervals) are shown for both data and model (with solid gray lines and dashed black lines, respectively). 
E4 1 hard

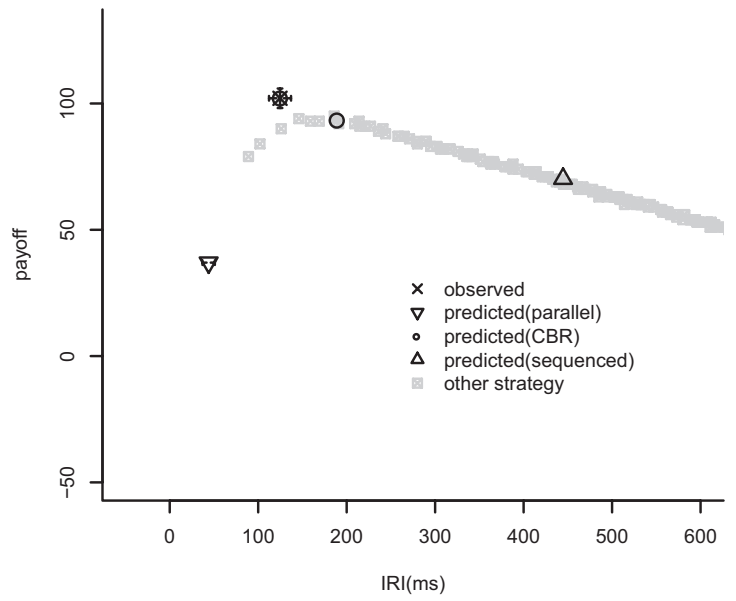

E4 3 hard



E4 5 hard

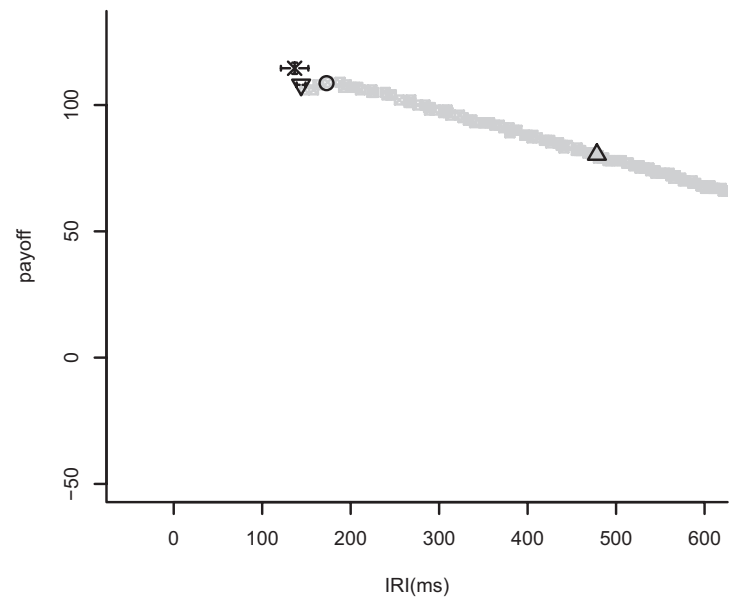

E4 2 hard

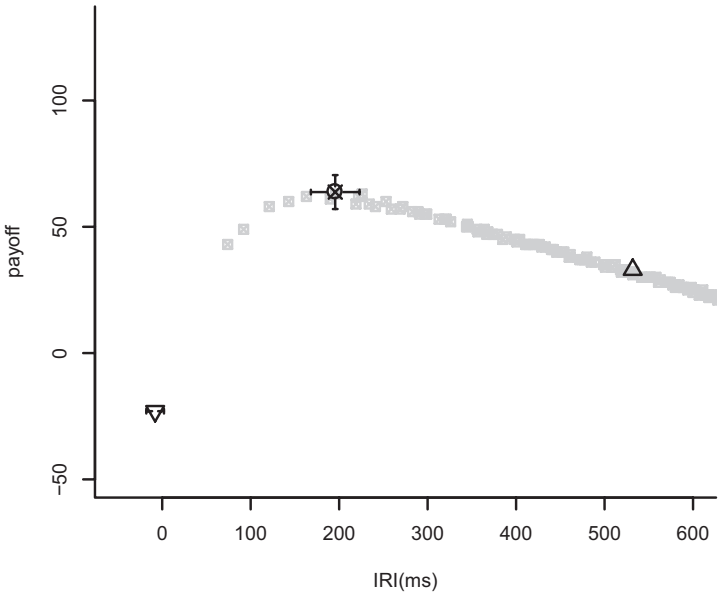

E4 4 hard

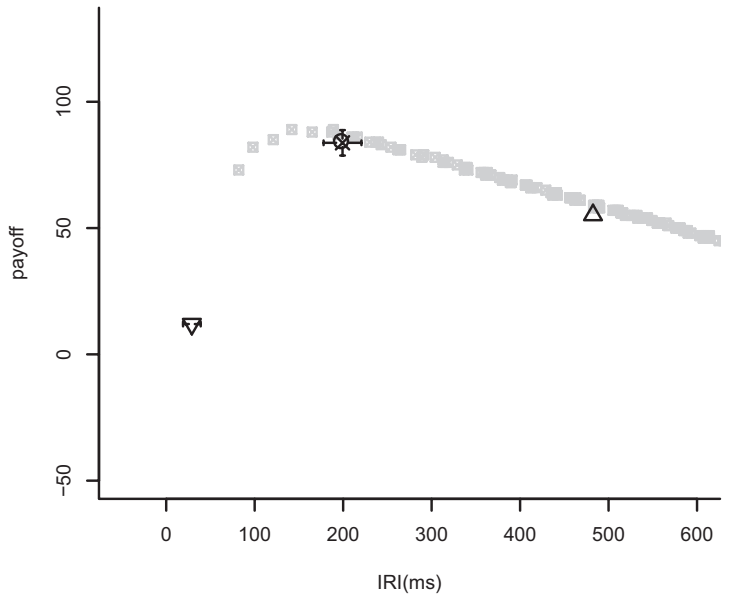

E4 6 hard

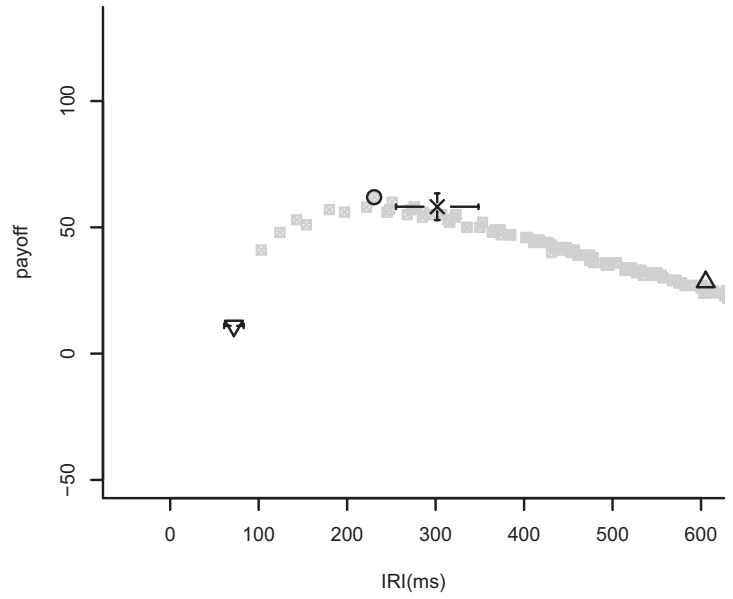

Figure 8 . Cognitively bounded rational (CBR) ordered response theory predictions of payoff against interresponse interval (IRI) for all 6 participants of Schumacher et al.'s (1999) Experiment (E) 4 with hard Task 2 at 50-ms stimulus onset asynchrony (SOA). 
systematic relationship to variance at lower SOAs as assumed by the model. A second important way that this prediction could fail to hold is that the specification of the strategy space is wrong. For example, the specified plausible space might be incomplete in some way that overlooks a critical subset of possible strategies.

There are six panels in Figure 8, one for each participant. In four out of the six cases the data lie on the payoff curve. The exceptions are E4 1 hard and E4 5 hard. This comparison provides some additional support for the ORT, to the extent that it indicates that the theoretically derived payoff space is consistent with the observed data.

It is also worth observing that there is some variation in the positioning of the peak of the payoff curve, consistent with the varying shape of the PRP curves presented earlier. For some participants, the best payoff was only just above 50 points, and for others, it was over 100 points. For some, the best IRI was about $100 \mathrm{~ms}$, while for others, it was over $300 \mathrm{~ms}$. These data suggest that accurate prediction of IRI required adaptation to individual RTs and levels of variance. Indeed, the peak of each individual payoff curve corresponds to the best strategy for the individual, not the best strategy for the sample.

In order to determine the quality of the IRI prediction across participants for all four experiments, we plotted mean payoff against mean IRI. In Figure 9 there are eight panels, one for each condition (easy-hard) of each experiment. Each mean point is plotted along with the between-participants $95 \%$ confidence interval. Each panel offers predictions, with $95 \%$ confidence intervals, from the cognitively bounded model and the two models that serve as our bound for a plausible range of behaviors: the parallel model and the serial model. Finally, payoff curves for the cognitively bounded model are plotted. The payoff curves represent the mean payoff across participants given each level of IRI within the strategy space. There are multiple payoff curves in each panel because there were multiple dimensions of strategic variation. One curve may, for example, represent a model in which the selection process of Task 2 was deferred until after the motor preparation stage of Task 1 and another may represent a model in which only Task 2 motor processing was deferred (see the Appendix).

It is important to note that the peak of each payoff curve is not the prediction of the model, though it may in some cases correspond. The prediction of the model is determined by taking the mean performance of the best strategies for each participant, whereas the payoff curve represents the mean performance of each strategy. Because participants did not always select the same strategy, the mean of the best (the prediction) is different from the best of the means.

Figure 9 provides evidence to support the cognitively bounded theory. First note that the parallel and serial models can be rejected. None of the data are in the prediction intervals of these theories. Focusing again on the E4 plots, which offer a critical test of the theory owing to the absence of resource conflicts, it can be seen that the data are within the prediction interval of the ORT model for both easy and hard tasks. E4 therefore offers strong support for the cognitively bounded theory of ordered responses. E1-E3 offer some further, though weaker, support. The most serious departures from predicted values occur in the hard conditions of E2 and E3, which suggests that the architectural assumptions of EPIC-V concerning the duration of response selection relative to perceptual processing might need to be modified, or a response selection bottleneck introduced (we take up the latter possibility below).

\section{Subadditivity}

In our earlier analysis of ACT-R, we observed that, under the strategy space implicitly explored by Byrne and Anderson (2001), ACT-R can predict both the presence and absence of subadditivity effects. We now turn to the present models, asking two questions. First, do the models systematically predict subadditivity effects? Second, how do the sizes of the predicted effects compare to the empirical values?

We calculated the mean subadditivity effects across the models of individual participants. These are presented, with the betweenparticipants $95 \%$ confidence intervals, alongside the observed subadditivity, in Table 2 . The subadditivity predictions for all four experiments are too large. The model tends to predict that the difficulty effect at short SOA is smaller than observed. In other words (as we saw in Figure 7), there is a tendency for the model to underpredict hard Task 2 performance and therefore overpredict subadditivity. Overprediction of subadditivity suggests that the theory lacks constraint and that too much strategic flexibility is available.

\section{Exploring the Implications of the Architectural Theory: Varying the Selection Bottleneck and Motor Jamming Assumptions}

One of the key aims of CBR analysis is to provide a way for data to discriminate between the predictions of strategically flexible architectures. We now present the results from explorations of assumptions concerning internal resource limitations: the motor jamming assumption and the no-selection bottleneck assumption. More specifically, we compare the results of three models. The models are (a) a no-selection bottleneck model with motor jamming (the model described above), (b) a model with a selection bottleneck and motor jamming (similar to ACT-R), and (c) a model with neither motor jamming nor a bottleneck. Table 1 provides a summary of the correspondence between each of the three models and the data from the 30 participants in the four experiments, in terms of mean RMSE and $R^{2}$.

\section{Is the Assumption of Motor Jamming Critical for Accounting for the Data?}

We tested the role of the motor response preparation bottleneck (motor jamming) in explaining performance in E1, E2, and E3 by deriving the predictions of models with and without the jamming assumption. (The assumption of motor jamming plays no role in E4 because the cross-modal design of the experiment specifically avoided modality conflicts.)

A two-tailed paired $t$ test of the mean square errors (MSEs) showed that the difference between the jamming and the no jamming model across the 30 participants was significant $(t=$ $-3.910, p=.0005)$. The architecture without a jamming assumption offered different predictions than the one with jamming. The results showed that for E1-E3, adaptation to system variance alone (i.e., eliminating the jamming constraint) offers worse predictions of SOA on Task 2 performance than the model with jamming $\left(R^{2}\right.$ 
E1 easy

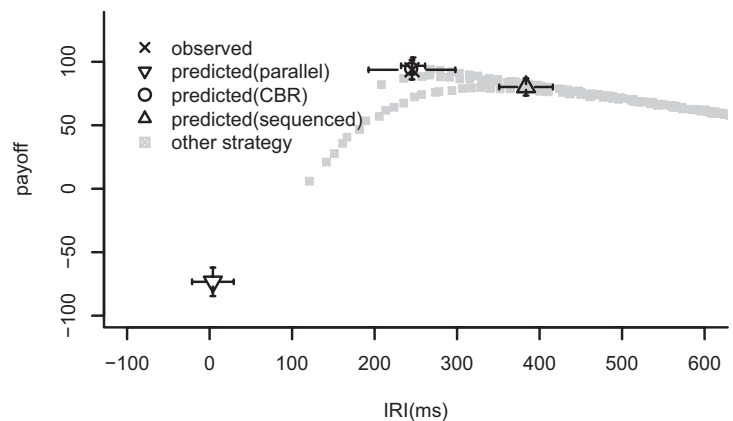

E2 easy

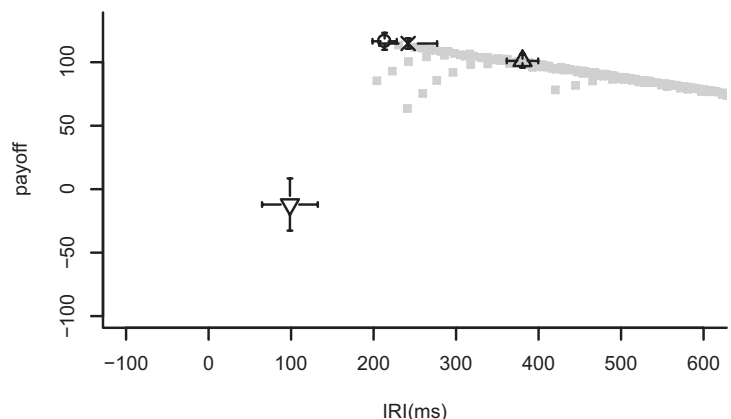

E3 easy

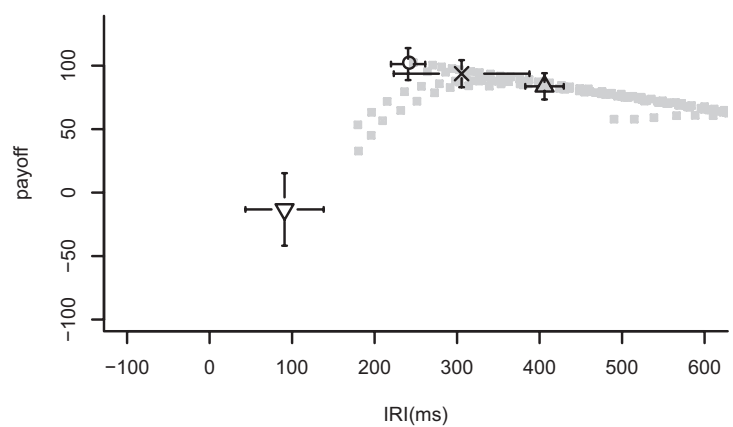

E4 easy

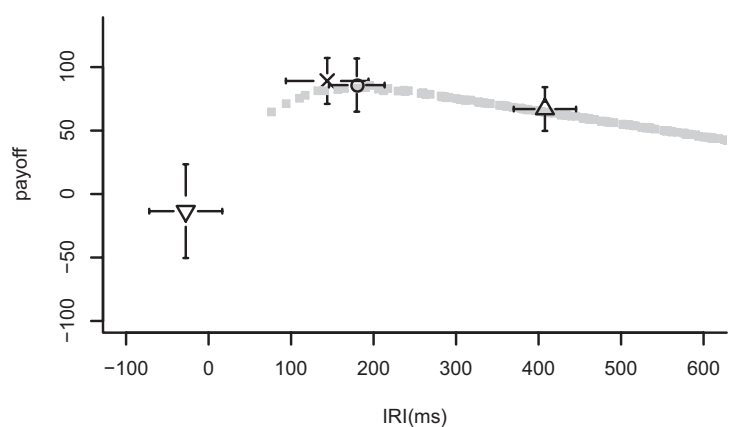

E1 hard

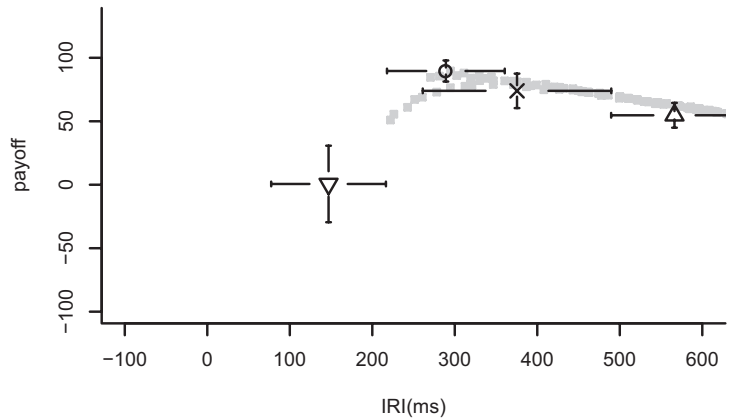

E2 hard

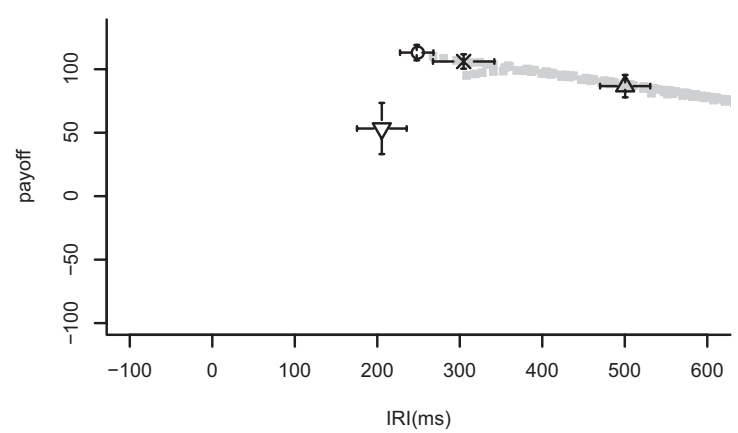

E3 hard

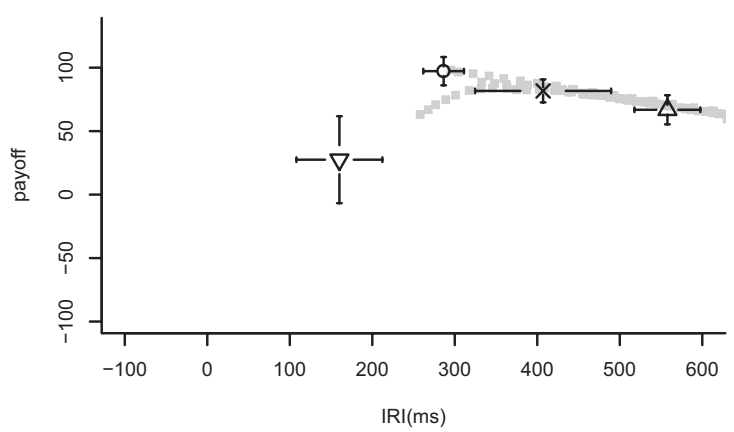

E4 hard

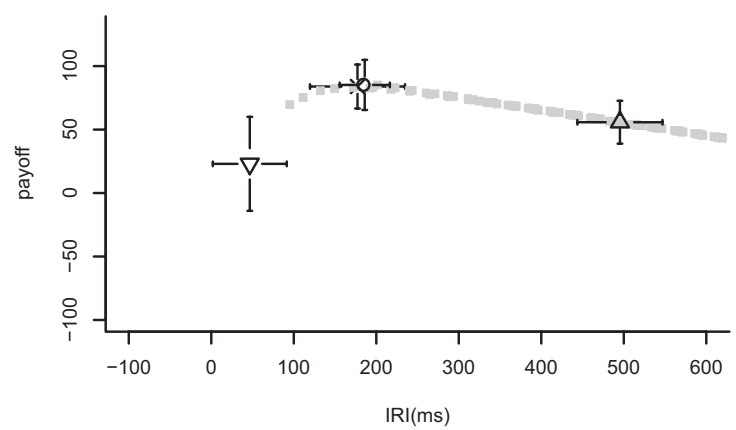

Figure 9. Cognitively bounded rational (CBR) ordered response theory predictions of payoff against interresponse interval (IRI) for easy and hard Task 2 conditions of all four experiments. The observed data are reanalyzed from Schumacher et al.'s (1999) Experiments (E) 1-4. The 95\% CIs (confidence intervals) are shown for both data and models. 
Table 2

Subbadditivity Effects (in Milliseconds) for Data and Cognitively Bounded Rational Model of Response Ordering

\begin{tabular}{lrrr}
\hline & & \multicolumn{2}{c}{$95 \%$ confidence } \\
interval
\end{tabular}

Note. Data are from Schumacher et al.'s (1999) Experiments 1-4.

values, reported in Table 1 , were $0.88,0.75,0.72$, and 0.85 , respectively; RMSE values were 67, 46, 93, and 41, respectively). The values of $R^{2}$ are lower, and the RMSE values are higher. Also, the data were not in the confidence interval of the model without jamming for either the easy or hard conditions of E1, E2, or E3. According to this criterion, the model predicted only two of the eight conditions across the four experiments.

The assumption that people adapt response timing to internal system noise is therefore not sufficient, on its own, to explain performance in E1-E3. Our analysis provides evidence that noise works in combination with Meyer and Kieras's (1997a) withinmodality, process jamming assumption to constrain adaptation. Jamming causes conflicts between the last 200 ms of Task 1 and the last $150 \mathrm{~ms}$ of Task 2 .

\section{Is the Assumption of No-Selection Bottleneck Critical for Accounting for the Data?}

In order to assess the role of the no-bottleneck assumptions, we tested ORT with an architecture that included a response selection bottleneck. Not only is a bottleneck theory a plausible account of the data supported (e.g., by Byrne and Anderson's, 2001, dual arithmetic experiments), but in addition it is likely to slow hard condition RTs more than easy condition RTs. It may therefore address the deficits of the no-bottleneck account evident in the hard condition panels of E2 and E3 identified above (see Figure 7).

In a bottleneck account, the two select processes competed for resource. In accordance with the instructional regime, Task 1 was prioritized, and as a consequence Task 2 selection was delayed. Task 2 selection could not start until after Task 1 selection had completed (the cognitive resource here operates as a queue). All other assumptions in the model were identical to the no-bottleneck model explored above, and no parameter values were changed.

On mean the selection bottleneck architecture offered higher $R^{2}$ and lower RMSE values than the no-bottleneck architecture for all four experiments (see Table 1). The data were in the confidence interval of the selection bottleneck model for seven out of the eight conditions, whereas the no-bottleneck architecture did well on five out of the eight conditions. According to this criterion, the model failed to predict the hard condition of E3. A two-tailed paired $t$ test showed that the difference between the MSEs of the bottleneck and the no-bottleneck models across all 30 participants was significant $(t=-5.1717, p=1.574 \mathrm{e}-05)$. The architecture with a bottleneck assumption offered better predictions than the one without.

The predictions of the bottleneck model are illustrated in Figure 10, which shows mean RT as a function of SOA for Task 2 across all four experiments. There are eight panels, including an easy and a hard plot for each experiment. The data are shown with the between-participants 95\% confidence interval, and the model's aggregate prediction is the mean of the predictions for each participant - that is, the mean of the best strategies for each participant (where, again, best is defined in terms of payoff, not fit to data). Figure 10 should be contrasted to Figure 7.

\section{Further Exploration of the Bottleneck Jamming Model}

It is evident from the model comparisons reported above that the bottleneck jamming model made more accurate predictions of the observed human data than the other two architectures under test. Here we further explore these predictions and focus in particular on the subadditivity predictions. The subadditivity predictions of the bottleneck and no-bottleneck models are contrasted to the data in Figure 11. It is important to keep in mind, again, that the predictions are derived from the strategy that generated the highest payoff using a Monte Carlo simulation and given the calibrated architectures. The predictions are not the result of effort to maximize $R^{2}$ or any other measure of model-data match.

Figure 11 shows that the subadditivity predictions of the bottleneck model are closer to the data than those of the no-bottleneck model but that, despite this increased proximity, only the data for E4 are within the prediction interval of either model. Despite the fact that none of the data falls within the prediction interval of the no-bottleneck model and one data point falls within the prediction interval of the bottleneck model, the difference between the subadditivity predictions of the two models is small.

\section{Discussion of the PRP Models}

We focus in this section on the implications of the above analysis for (a) theories of the cognitive architecture and (b) ORT. We then make brief comments on the specifics of the analysis method. The implications of the use of CBR analysis for cognitive science in general are addressed in the General Discussion.

\section{Implications for Theories of the Cognitive Architecture}

We believe that the current analyses provide the strongest evidence to date for the following conclusions:

1. In the ordered PRP task, performance is an adaptation to multiple architectural constraints that include at least noise and motor process interference. No other existing account is able to give quantitative explanations of individual behaviors that range from nearly perfect time sharing to rather steep PRP curves, and this quantitative account may be improved by further assuming the constraint of a selection bottleneck (see Conclusion 5 below). 
E1 easy

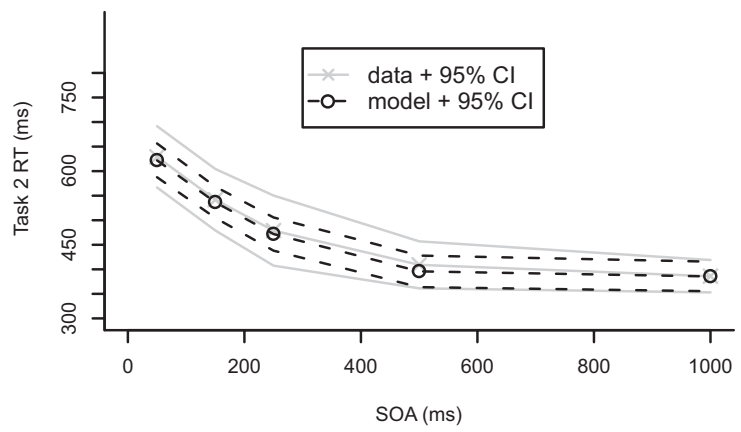

E2 easy

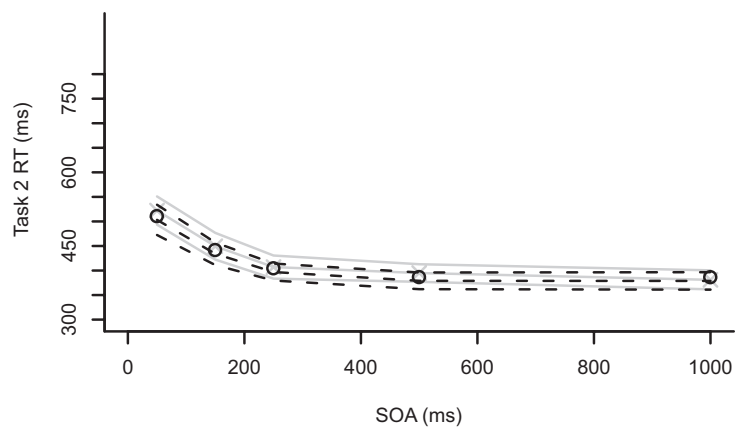

E3 easy

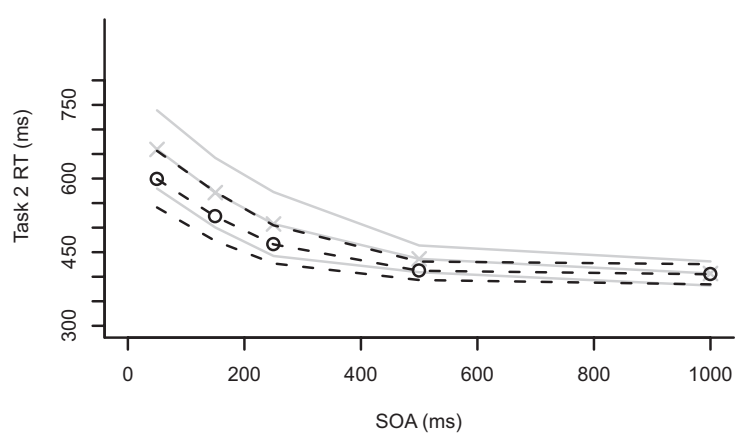

E4 easy

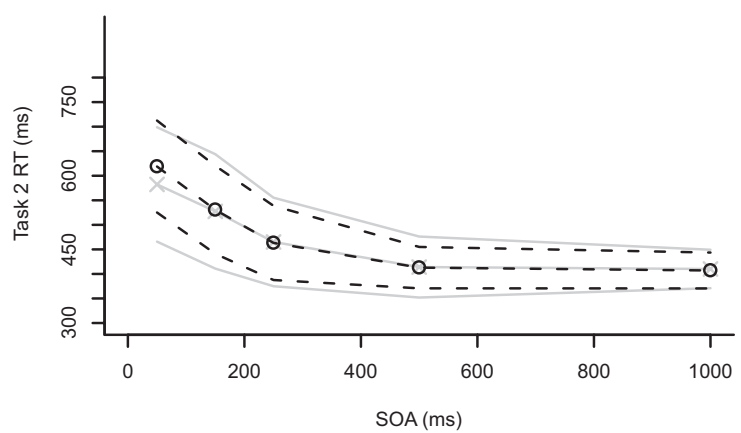

E1 hard

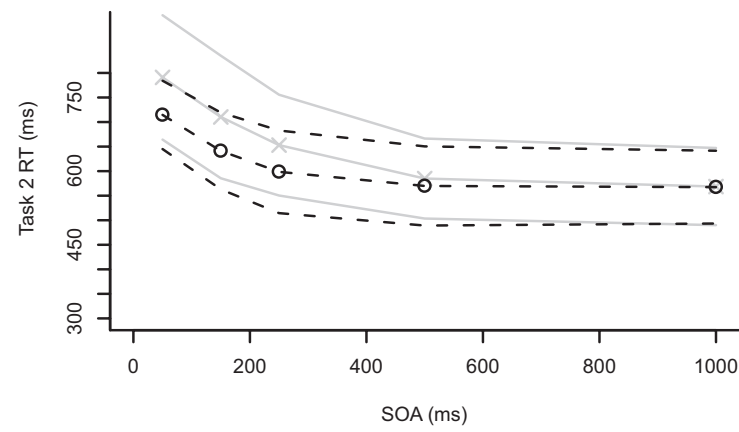

E2 hard

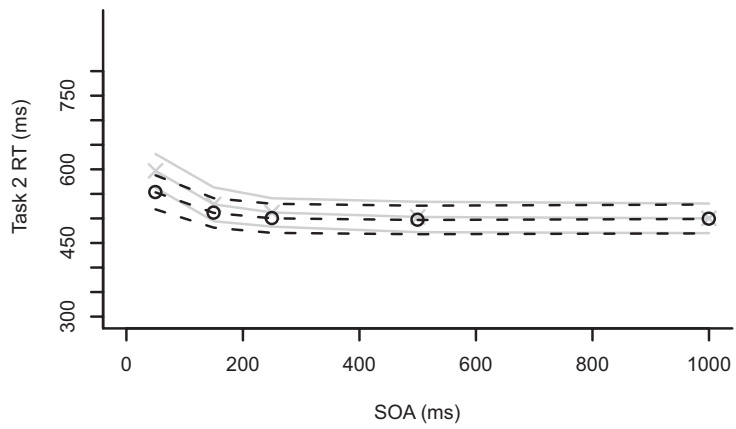

E3 hard

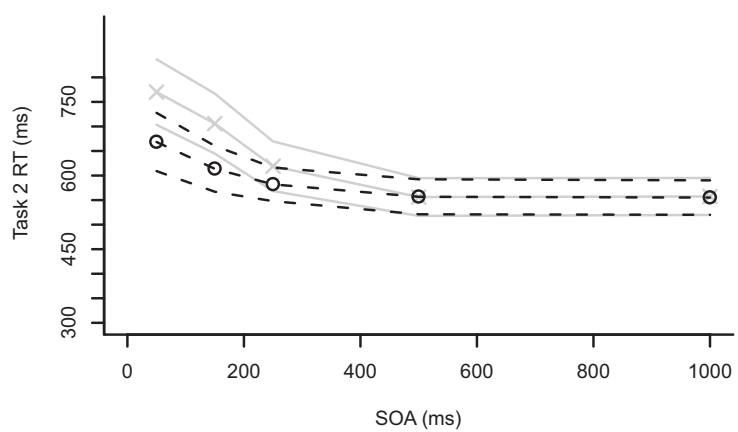

E4 hard

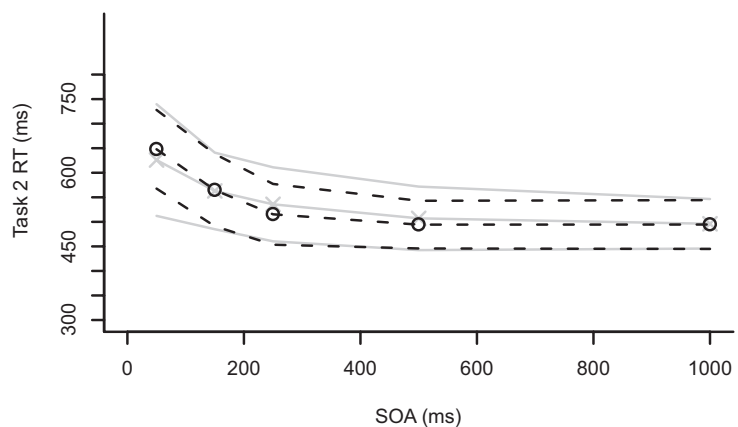

Figure 10. Bottleneck model predictions and mean human data for Task 2 response time (RT) as a function of stimulus onset asynchrony (SOA) and Task 2 difficulty (easy vs. hard). The data are reanalyzed from Schumacher et al. (1999)'s Experiments (E) 1-4. The 95\% CIs (confidence intervals) are shown for both data and model (with solid gray lines and dashed black lines, respectively). 


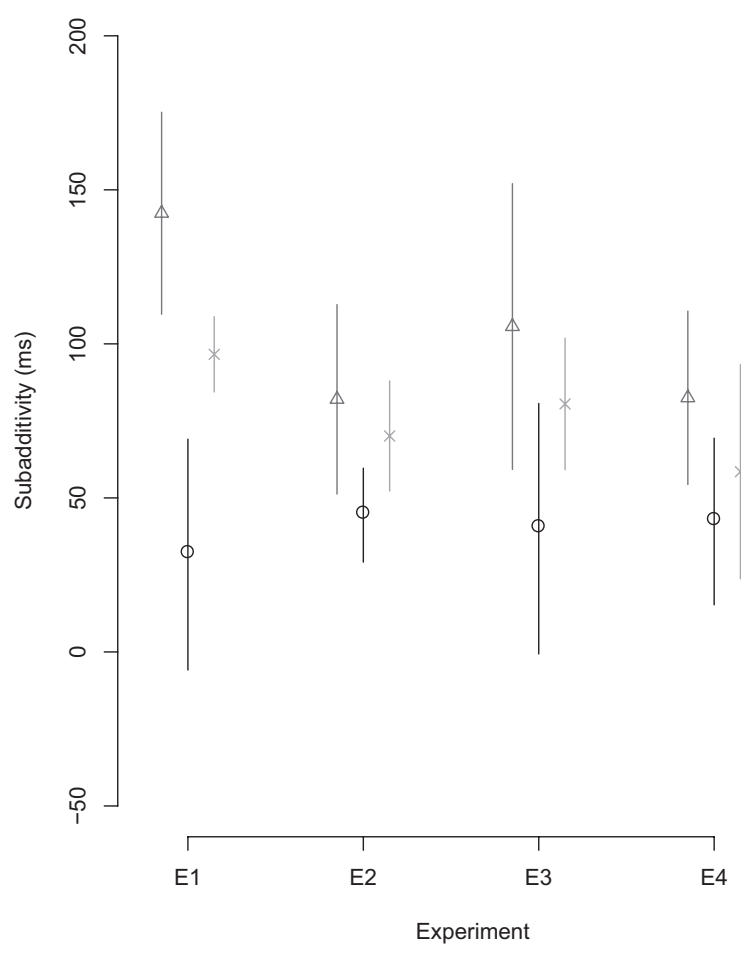

Figure 11. Subadditivity effects for models and observations. Shown are observed subadditivity (circles), no-bottleneck model subadditivity (triangles), and bottleneck subadditivity (crosses). Observations are from Schumacher et al. (1999)'s Experiments (E) 1-4. The 95\% CIs (confidence intervals) are shown for both data and models.

2. One of the primary constraints that shapes performance is noise. Individual performance can be predicted as adaptation to noise (see no bottleneck-no jamming model in Table 1). Although between-participants variance was explained only moderately well in one of the four experiments (E4), it was the experiment that provided the most direct test of adaptation to noise.

3. Another key constraint is motor process interference (jamming). The analysis provides evidence that jamming plays some role in constraining PRP performance.

4. There is some support for the control signals made available by the ACT-R and EPIC architectures for coordinating the Task 2 response. However, a systematic comparison of architectures with different subsets of control signals is yet to be done.

5. Perhaps surprisingly, carefully deployed PRP paradigms, such as that in Schumacher et al., 1999, are capable of distinguishing between architectures with and without response selection bottlenecks-but this discrimination is far more subtle than the original motivation of the paradigm would suggest. Under assumptions of Type C rationality, both kinds of architectures are capable of producing the classic PRP curve, and the gross shape of this curve is not determined by the presence or absence of the bottleneck. (Rather, it is determined by the adaptive response to noise.) Nevertheless, the bottleneck models were systematically and reliably better at accounting for the data across all four experiments. We emphasize again that this conclusion is relative to the specific auxiliary assumptions that we adopted for the EPIC-V architecture. A more comprehensive evaluation of the role of the bottleneck assumption would require an even larger scale modeling effort that evaluated the effect of the bottleneck assumption against a space of plausible auxiliary assumptions.

\section{Implications for ORT}

None of the architectural variants were sufficient to explain the data without the additional assumption that people adapted strategically. We defined ORT as stating that any two responses that must be ordered will be coordinated in a way that maximizes subjective expected utility. In standard tasks where both accuracy and speed are important, utility is increased by smaller temporal separations but decreased by reversal errors. Elaborated with the auxiliary architectural assumptions, ORT provided precise quantitative predictions.

ORT was tested against data from PRP experiments reported by Schumacher et al. (1999), and we found that the data offer strong support for the theory. We showed that incremented with existing architectural assumptions, a selection bottleneck, and gammadistributed noise, the strategic adaptation inherent to ORT predicted the quantitative relationship between RT2 and SOA at short SOA in seven out of eight conditions across four experiments (see Figure 10). Furthermore, this relationship, captured by the shape of the PRP curve, varied significantly and systematically between participants and accounted for some of this individual variation. We also showed that the IRIs observed in the 6 participants of Schumacher et al.'s (1999) E4 are partially captured by the theory. E4, unlike Schumacher et al.'s (1999) other experiments, provides a critical test of the assumption that people adapt to internal variance because there are no resource conflicts between Task 1 and Task 2, and it is therefore possible that any slowing of the Task 2 response is entirely strategic. In short, the correspondence between the predictions and data for Experiment 4 provide strong support for the claim that the PRP curve, in its details, is the result of participants adapting nearly perfectly to the payoff regime and their own system noise.

Our analysis of Schumacher et al.'s (1999) E1-E3 offers further, but weaker, support for ORT. The hard condition IRI predictions do not achieve the high level of quantitative prediction of the data seen with E4. There are two points to note about these results. First, to the extent that ORT does predict performance in E1-E3, it does so because of the assumption that people will adapt optimally to the entire set of constraints, including the motor jamming constraint and the selection bottleneck, not just response variance. Second, on the basis of the method that we have proposed (see A General Approach to Understanding Adaptation to Task and Architecture section), it is clearly possible for a theory of the human cognitive architecture to fail to predict the data.

One reason for the differences between the ORT predictions and Schumacher et al.'s (1999) data for E1-E3 may be that some participants failed to approach the performance asymptote (i.e., 
participants may not have had sufficient opportunity to adapt). Participants appear to improve between the last two sessions in E2 and E3. In contrast, participants appear to be closer to asymptote in E4, as Session 3 RTs are no faster than Session 2 RTs. It may also have been due to incorrect constraints in the architecture. It is possible, for example, that the relative values of the default durations are incorrect. It is likely that if, with the bottleneck architecture, we had used shorter defaults for perception and/or longer defaults for selection, then there would have been a greater effect of SOA on Task 2 hard RTs.

We believe that these conclusions represent a significant advance in researchers' understanding of how humans cope with a certain class of multitasking situations. These results encompass quite general conclusions about the impressive adaptiveness of humans in these situations and the (individually varying) processing constraints that bound that adaptation as well as conclusions concerning the details of the strategic adaptation and implicated processing architecture. These conclusions rest on results made possible by CBR analyses.

\section{Comments on the Use of CBR Analysis}

An important property of the analyses above is that quantitative predictions were not obtained by adjusting free parameters in order to increase the fit between model and data. We determined the architectural parameters of the model by theoretically driven calibration, not by maximizing fit to the outcome variables (RT at 50-ms SOA). We determined the selected strategy and its corresponding quantitative deferment time by optimizing a payoff function, again, not by maximizing data fit. There were no other parameters. It is on this basis that we claim that the predictions made for E4 and illustrated in Figures 6, 7, 8, and 9 represent predictions of the architecture plus the assumption of cognitively bounded rationality, and not of the analyst or of the strategy. They are predictions that suggest that the theory offers a quantitative explanation of the data.

One analytic device that we used to test and understand the predictions was the payoff curve (see Figures 8 and 9). The data gave us point estimates of the payoff achieved by each participant against their selected IRI. The models predict an inverted U-shaped relationship between payoff and IRI. Using these curves, we made three evaluations of the theory: (a) Did the prediction interval corresponding to the peak of the payoff curve for each individual participant correspond to the participants performance? (b) Did the prediction interval corresponding to the betweenparticipants mean of the peak of the payoff curves correspond to the mean participant performance? (c) If performance was not predicted by the peak of the curve, was it at least predicted by the curve? These three uses of the payoff curve allowed us to establish where the data supported the theory and where they did not.

In addition, the payoff plots made explicit the shape of the utility function given the theory of cognition and the strategy space. They thereby made it possible for us to determine that there was, for most participants, a defined peak to the curve-which was not necessarily the case-and that the risks associated with going fast were much greater, owing to the steepness of the curve, than the risks of going slow. This suggests that it is riskier to approach the peak from the faster part of the curve, and therefore that when participants are not at the peak, they will tend to be on the slower part. In fact, of the 28 participants across the 4 experiments who failed to reach optimal performance, 20 were clearly on the shallower side of the payoff curve.

\section{General Discussion}

We have argued that the extreme flexibility of human cognition poses a particular challenge to cognitive science. The fact that people can and do exhibit strategic flexibility even on very simple tasks makes it difficult to determine the contribution to behavior of the invariant mechanisms of cognition. Moreover, we have shown that the challenge has been made more difficult by the use of model fitting to test strategically flexible architectural theories of cognition. We have demonstrated that the intuitive sampling of the space of possible strategies, evident in attempts to explain data using model fitting, does not provide a sound basis for claiming that particular architectural theories explain and predict the data and, also, that it significantly compromises weaker demonstrations of consistency (see Summary of the Analysis of the ACT-R Models section).

In response we have argued that to make progress on a scientific understanding of the invariant mechanisms of cognition, researchers must find ways of inferring which of the range of possible strategies is rational given the constraints. We have proposed an approach to this problem that starts from the assumption that behavior is cognitively bounded rational and uses this assumption to select strategies. People are limited by cognition and perception. They are limited by the performance of their motor system, and they are limited by the characteristics of the task environment. People adapt within these limits to a utility function. Assuming that human adaptation is Type $\mathrm{C}$ rational allowed us to calculate the prediction of a mechanistic theory without recourse to intuitive strategy sampling or to fitting quantitative or strategic parameters to the target data to be explained. Assuming rationality, given constraints, allows the control of degrees of freedom that are otherwise introduced by permitting theories to exhibit strategic flexibility.

We demonstrated the usefulness of our approach, called CBR analysis, by calculating the predictions of three theories of the cognitive architecture thought to underpin PRP performance. The first had no-selection bottleneck (like EPIC), the second had a selection bottleneck (similar to that of ACT-R), and the third had no-selection bottleneck and no motor system jamming. Testing the theories with $\mathrm{CBR}$ analysis left open the possibility that one or more failed to predict the data. It happened that two (selection bottleneck and no-selection bottleneck, both with jamming) did not fail and that the bottleneck model offered significantly lower root-mean-square deviation than the no-selection bottleneck model. Moreover, a theory of the architecture that did not have within-modality motor system jamming failed to adequately account for the data, providing evidence that there is motor system interference and that people adapt to this interference in their attempt to satisfy task demands.

We also provided evidence to support ORT. Irrespective of the particular architectural assumptions (with or without a bottleneck, with or without jamming), the models predicted some slowing of RT2 at short SOAs. To the extent that participants demonstrated a similar slowing of RT2, there is quantitative evidence that it is due to a strategic response to subjective utility. 
In what follows, we first consider two related approaches in more detail (bounded rationality and theories of learning). We then consider potential critiques and limitations of the approach (critiques of the assumption that people can achieve optimal performance and whether our approach can be applied to understanding behavior before reaching the asymptote). Before concluding, we briefly draw out some general implications for cognitive science (the implications of auxiliary assumptions in understanding experiments, the importance of theories of subjective utility, and applications of psychological theory to design problems).

\section{Related Approaches}

In the introduction we identified some of the major ideas in cognitive science that led to CBR analysis. We now take up a brief but more detailed discussion of the relative costs and benefits of some of these approaches along with other closely related work.

\section{Bounded Rationality}

One view of CBR analysis might be that it is simply a formal approach to bounded rationality (Simon, 1955, 1992). Both approaches place an emphasis on understanding how constraints on human information processing bound rational behavior. There are instructive differences, however. Bounded rationality, as conceived and applied by Simon, is a framework for understanding a broad range of human behavior, in contexts ranging from the psychological laboratory to the complex environments of modern organizations (Simon, 1957, 1979, 1989, 1997). The explanatory power of the framework is evident in the astonishing range of contributions that Simon and his colleagues have made across several disciplines. We do not intend to advance CBR analysis in this article so broadly, but it is important to understand the differences in the approaches as applied to understanding relatively short-time scale behavior as typically observed in a cognitive psychology experiment.

There are three key differences. First, to the extent that bounded rationality is rational, it focuses on decision making, either the sequential decision making that unfolds over seconds in laboratory problem-solving tasks or the longer time-frame decision making that unfolds over days or longer in the economic world (Simon, 1997). In contrast, we have demonstrated that CBR analysis is relevant to understanding perceptual-cognitive-motor coordinations that unfold in the 10s of milliseconds to seconds (which can also be viewed as a rapid series of control decisions). We have not investigated whether CBR analysis is useful in understanding decision-making tasks over longer time periods.

Second, to the extent that bounded rationality focuses on investigating the processing constraints that bound behavior, it has not emphasized the role of maximizing a utility function. For example, Simon's contributions to understanding short-term memory, longterm associative memory, and problem representations (e.g., see the compilations in Simon, 1979, 1989) were made without benefit of an explicit consideration of the effects of the utility functions that human participants might have adopted in the experimental situations. (These contributions also serve as a reminder that we are not, of course, arguing that progress cannot be made without utility function analyses.)
Third, to the extent that bounded rationality focuses on investigating the nature of the variable strategies that humans use to accomplish their goals, it has done so via more direct observation and analysis of strategies, rather than formal analysis of the adaptive qualities of strategy spaces. Examples include Newell and Simon's (1972) classic work on problem solving. Newell and Simon were clear that the observed strategies were shaped by quite general invariants of human information processing (e.g., limited short-term memory), but the theory was not used to generate predictions of the details of the strategies in advance. Rather, the major advance of the classic problem-graph analyses was that they showed how relatively long stretches of complex cognitive behavior could be understood as arising from systematic explorations of a problem space formulated by the individual.

It is instructive to briefly consider how the Newell and Simon (1972) approach to problem solving might be related to CBR analysis. The problem space theory of human problem solving can be seen as taking the necessary first step toward such an analysis, because it helps to define the space of possible strategies (i.e., the space of possible ways of searching the problem space). But CBR analysis opens up the possibility for more deeply explanatory theories of the strategies observed in problem-solving behavior. Such theories would make stronger connections between detailed assumptions about the constraints imposed by cognitive architecture and the payoffs imposed by the local task environment. They could be put to stringent empirical test by showing how strategic variation arises from variation in individual processing constraint (as we have done in the PRP analyses above) or by variation in external task payoff manipulated in controlled fashion (as was done in SDT studies; e.g., Tanner \& Swets, 1954; and as we have done in unpublished work on response ordering). For example, researchers using such analyses might attempt to formally derive classes of methods such as iterative deepening (Newell \& Simon, 1972) as the optimal response to limited working memory but furthermore show that the precise nature of such methods depends on the specific speed-accuracy tradeoff imposed on the problem solver and the nature of their individual (and individually calibrated) processing constraints.

\section{Theories of Learning and Task Acquisition}

The mechanisms by which novice performance is transformed into expertise is a major source of constraint on behavior (e.g., Newell \& Rosenbloom, 1981; Ohlsson, 1996). One response to our work might be to wonder why it is worth using optimization: Why not simply articulate theories of the learning mechanisms (i.e., theories of the trajectories from novice to expert behavior)? We of course advocate the continued development of such theories-our aim here is to point out some weakness of such an approach relative to CBR analyses.

There are two problems. First, to the extent that there is in fact a correspondence between optimally derived behavior and observed asymptotic behavior, then such correspondence renders the specifics of the learning theory irrelevant to the understanding of asymptotic behavior-all that matters is that perfect adaptation is possible. This does not rule out using alternative learning mechanisms as a means of deriving the optimal behavior, but in this view their selection would be based primarily on considerations of 
practical computational properties, rather than on consideration of cognitive theory.

A second problem is that while there is no reason to believe that progress on learning is any slower than on theories of any other aspect of cognition, there are no sufficiently well-developed theories of learning that are comprehensive and robust enough to be set as a constant in a framework designed to test theories of other aspects of the architecture. It is important to understand the significant computational, theoretical, and empirical demands that a learning theory must meet to serve the role of a general basis for CBR analyses: (a) It must provide the means to transform some declarative form of the task specification into procedural behavior-essentially provide a theory of instruction taking; (b) it must be fully computationally realized; and (c) it must have sufficient independent empirical and theoretical support that departures in the predictions can be unambiguously attributed to other processing constraints under test, and not to the underlying learning theory.

The recent impressive advances in instruction taking in ACT-R provide examples of learning theories that begin to meet some of these constraints, but these models are not yet general enough and robust enough to serve as the basis for a broad approach. It is worth noting, however, that the grounding of the ACT-R approach in reinforcement learning (Sutton \& Barto, 1998) is a promising direction. Various mechanisms for reinforcement learning are well specified and computationally well understood, and have the virtue of providing a theory of the learning of control, precisely what is needed to yield strategic adaptation. The promise of reinforcement learning as the basis of CBR analyses can be seen in other recent work as well (e.g., Reichle \& Laurent, 2006).

\section{Potential Critiques and Limitations of the Approach}

\section{People Are Suboptimal}

One response to the approach proposed in the current article is that "people are not optimal." Given the controversy over the use of optimality criteria in understanding cognition (e.g., Gigerenzer \& Todd, 1999; Simon, 1992), this objection needs to be taken seriously. Our response has several parts (for further discussion of the issue, see Howes et al., 2007; Sperling \& Dosher, 1986; and Swets et al., 1961).

1. It is important to identify what kind of rationality is being denied with the claim that people are not optimal. In particular, we distinguish normative rationality (Type $\mathrm{N}$ rationality) from cognitively bounded rationality (Type $\mathrm{C}$ rationality) - the assumption that behavior can become optimal, through learning, given constraints imposed by the local task environment and by psychological mechanisms. It is certainly the case that it is often difficult for people to conform to Type $\mathrm{N}$ rationality. In contrast, our focus, in the tradition of SDT (e.g., Sperling \& Dosher, 1986), is on optimal adaptations relative to both the internal and the external constraints.

2. But even when people focus on Type $\mathrm{C}$ rationality, there are many reasons why they may not develop Type C optimal solutions. An important limiting factor is that such solutions may not be discoverable given some re- stricted experience with the task environment (e.g., see Fu \& Gray, 2006). Also, it may be the case that only some task environments have discoverable maxima because of the nature of the multidimensional payoff surface. It turned out that the PRP payoff curves in the Schumacher et al. (2001) experiments contained relatively clear maximum peaks, but this was by no means necessary (in fact in other unpublished empirical work conducted in our labs, we are discovering that intuitively plausible payoff schemes do not always lead to such well-behaved payoff surfaces).

3. The PRP task is a member of an important class of tasks, utility learning tasks, that may be particularly well suited to optimality analysis. These are tasks for which substantial incremental feedback is provided over many hundreds, or thousands, of trials. Participants therefore have the opportunity to tune their response to the payoff function. Significant advances have been made by treating tasks that offer incremental feedback as opportunities for optimization (e.g., Bogacz et al., 2006; Gray \& BoehmDavis, 2000; Gray et al., 2006; Reichle \& Laurent, 2006; Sutton \& Barto, 1998).

\section{Understanding Behavior Before Reaching the Asymptote}

Given the emphasis on explaining behavior at the asymptote, a natural question to ask is, Does the approach have any value if the goal is to understand behavior early in practice or behaviors involving novel stimuli?

Let us begin to answer this question by observing that although there are a few cases of analyses of the first few trials of performance on some task (Goeree \& Holt, 2001; Keppel \& Underwood, 1962), in general, a focus on very early aspects of performance is relatively rare in cognitive psychology. The reason is clear: Performance is much more variable at this stage of task acquisition. Participants are still in the process of understanding what to do; they are still in the stage of exploring the strategy space and discovering their utilities. In short, most cognitive experiments usually involve both a practice stage and a fairly extensive set of trials during which performance begins to stabilize.

Furthermore, many experiments involve extensive practice on the task itself, with later performance measured on the same task but with novel stimuli. We believe this fairly conventional approach to empirical design lends itself well to the application of CBR analyses that encompass novel or unpracticed behaviors. The key is to focus on the idea that to make a prediction from a cognitive architecture it is imperative to respect the assumption that people make rational choices in response to a subjective utility function given the constraints, including the constraints imposed by the architecture. While it should be expected that people achieve lower utility earlier in practice than they will subsequently achieve, it is possible that the early level of performance can be considered rational given constraints that include the architecture but which also include the limited individual experience of the task environment.

For example, an attempt to use CBR analysis to explain SOA effects on early trials of PRP tasks should focus on an analysis of boundedly rational choice given each participant's limited knowl- 
edge of the response distributions (as acquired from previous trials) and given expectations of the remaining number of task trials. Here, CBR analysis makes contact with research on the exploration versus exploitation tradeoff and problems concerning the temporal discounting of future utility. For a recent review of some of these issues, see Cohen, McClure, and Yu (2007).

\section{The Scope of CBR Analysis}

In this article and in our previous work, we have demonstrated that CBR analysis is applicable to a narrow range of tasks. These are tasks that, as we have said, involve a tight coordination between perceptual, cognitive, and motor processes. The dual-task paradigm explored in the current article is one example, but others have included applied tasks extended over longer time periods, for example, multitasking while driving and cockpit control tasks (Eng et al., 2006; Brumby, Howes, \& Salvucci, 2007; Smith, Lewis, Howes, Chu, \& Green, 2008; Tollinger et al., 2005). Eng et al. (2006) used the framework to help understand adaptations to the order of subtasks in a cockpit control task. Brumby et al. (2007) used the framework to help understand strategies for interleaving dual tasks during an ongoing dynamic task. Smith et al. (2008) used the framework to understand how individual differences in attentional switch costs would yield different optimal strategies for using a database lookup interface. In these cases the intention was to understand the implications of an interface design for behavior. While these tasks take durations from under $500 \mathrm{~ms}$ to over $5 \mathrm{~s}$ to complete, they all share the property that the space of possible adaptation concerns scheduling (i.e., when to perform one subtask given the temporal properties of the others; Schweickert, 1980). A potential critique of CBR analysis is therefore that it is limited to this relatively narrow range of tasks.

Although our demonstrations are narrowly scoped, we believe that CBR analysis has lessons for studies of some of the broader range of tasks used to provide evidence for theories of the human cognitive architecture, including perhaps tasks that involve the use of working memory in immediate behavior (Gray et al., 2006), task switching (e.g., Altmann \& Gray, 2008), probability learning (Shanks, Tunney, \& McCarthy, 2002), and perhaps problem solving (Fu \& Gray, 2006).

For an example, consider the working memory task studied by Gray et al. (2006). The task is to reproduce, in a workspace window, a pattern of eight randomly arranged colored blocks displayed in a target window. Every time that a participant looks at the target window, they can choose to encode between one and eight blocks in memory. They also incur a small time cost. If an individual chooses to encode one block on each visit, then a time cost of eight units will be incurred, but that individual will also be exposed to a very low risk of forgetting which blocks are where. If in contrast, a person chooses to encode eight blocks on a visit, and they do it successfully, then they will only incur a single time cost unit, however there is a much greater risk of forgetting one or more of the items and therefore needing to make a revisit. Gray et al. (2006) found that participants encoded a larger number of items on each visit when the incurred time cost of moving to the target window was higher due to a delay enforced by the computer software. Delays of 3,200 ms encouraged people to encode more in memory on each visit than delays of 0 ms. Gray et al.'s (2006) findings support the conclusion that people adapt the use of mem- ory to the time costs of action and therefore to utility (see also Smith et al., 2008).

The problem for participants in Gray et al.'s (2006) study is to choose a strategy rationally given the bounds on cognition (memory) and the bounds on the task environment (the time cost of accessing the target environment). Therefore, as the problem is to choose an encoding strategy that maximizes utility given the constraints imposed by the cognitive architecture, it might be the case that CBR analysis could be used to make predictions about participant performance. The key element required in an empirical study would be data that provide a means of calibrating a theory of working memory (e.g., ACT-R's activation-based theory) to individual capacity limits. Such a calibration, combined with the temporal calibration described in the current article and with a utility function, would make a CBR analysis possible.

\section{Additional Implications for Cognitive Science}

Although we view the work reported in this article as placed firmly within the tradition of computational approaches to understanding cognition (Anderson, 2007; Meyer \& Kieras, 1997a; Newell, 1990) and as building on several major theoretical approaches in cognitive science, we nevertheless believe there are significant implications of this work for future directions in cognitive science.

To further understand these implications, we briefly explore here two topics: (a) crucial experiments and auxiliary assumptions and (b) the subjective utility function.

\section{Crucial Experiments and Auxiliary Assumptions}

A crucial experiment, for example, as described by Lloyd (1999, p. 214), would be one that not only provided positive support for the theory under test but which also supported the rejection of alternative accounts. In an ideal world, PRP experiments would have this property. A selection bottleneck theorist might design an experiment that supported a selection bottleneck theory and also provide evidence against a strategic adaptation to PRP tasks. Similarly, a no-selection bottleneck theorist might hope that subadditivity supported parallel cognition and also supported the rejection of a selection bottleneck account of PRP effects. The reality, according to the Duhem-Quine thesis (Harding, 1976), is that no single empirical observation will be sufficient to reject alternative accounts.

According to the Duhem-Quine thesis, one reason that it is difficult to design crucial experiments is that an empirical test of a hypothesis requires one or more background assumptions (also called auxiliary assumptions or auxiliary hypotheses). The hypothesis in question does not by itself make predictions; rather the consequences of the hypothesis typically rest on a combination of theory and background assumptions. This fact prevents a theory (e.g., that cognition is subject to a selection bottleneck) from becoming conclusively falsified through empirical means if the background assumptions (e.g., strategic adaptation and other architectural constraints including motor system interference) are not proven.

One response to this problem, perhaps the modal response in psychological research, is to explore many variations of a paradigm in a search for convergent evidence. If one experiment is not 
sufficient, then do many and in each try to expose the consequences of one more of the auxiliary assumptions. We might, for example, change the design of a PRP experiment so that participants are not instructed to order the two responses. An observed PRP effect under these conditions is less likely, the reasoning goes, to be the consequence of strategic adaptation to an ordering instruction.

In our view, however, varying a paradigm is an essential but ultimately insufficient response to the problem of auxiliary assumptions. According to the analysis that we have presented above, it is important to understand the space of possible adaptations within a paradigm in order to understand the implications of the associated findings and to thereby make informed decisions about what paradigm variations might be useful. If strategic flexibility is ignored and unfounded assumptions are made about which strategy people adopt, then otherwise sound reasoning about the implications of a particular assumption will be undermined.

We summarize our position about crucial experiments as follows. Although it is tempting to assume that some new paradigm will addresses the strategic flexibility problem and will provide the crucial resolution to a particular theoretical debate, we are not optimistic about the analyst's ability to clearly see the implications of adaptation unassisted by the kinds of formal methods exemplified by CBR analysis. This circumspect approach to strategic variability is consistent with the views expressed by a number of cognitive psychologists, who, after reviews of a range of influential paradigms (each in a different subfield), concluded that strategic considerations must take a prominent role in the analysis of data (Forster, 1979; Hansberger, Schunn, \& Holt, 2006; Lohse \& Johnson, 1996; Meyer \& Kieras, 1997a, 1997b; Newell, 1973, 1990; Payne, Howes, \& Reader, 2001; Payne, Richardson, Howes, 2000; Schunn \& Reder, 2001; Siegler, 1994, 1999; Sperling \& Dosher, 1986).

\section{The Subjective Utility Function}

Another important and related methodological implication of CBR analysis is that modelers must pay more attention to the utility function or, more precisely, to the subjective utility function adopted by participants in experiments concerning immediate behavior. For the purposes of the analysis presented in the current article, we have assumed that the subjective utility function is determined by the experimental instructions, but the reality is probably more complex (Kieras \& Meyer, 2000). Participants presumably trade off a desire to contribute productively to the experiment, and the desire for cash bonuses, with other motivational factors, such as a desire to get out of the lab quickly or to exert minimal effort. Motivational factors external to the instructed payoff regime are likely to be significant and may interact with the perceived value of individual strategies. In addition, little is known about the relative weight that people give to, say, minimizing time, versus minimizing memory, versus minimizing optional instructional policies (such as those used by Schumacher et al., 1999).

Some recent work does advance assertions about what people are trying to do (e.g., the soft-constraints hypothesis of Gray et al., 2006). However, contrary to this hypothesis, the work that we have reported in the current article suggests that at the sub-3-s level, people who are given a payoff regime may neither be biased by a desire to minimize memory (or at least resources) nor be biased by a desire to minimize time. Instead, most select a processing schedule that optimizes according to the externally imposed instructional criteria (see A Cognitively Bounded Theory of Ordered Responses section).

More research is needed on this issue. In general, we expect that it is difficult to calculate the predictions of a theory of the human cognitive architecture without a complementary theory of the subjective utility function.

\section{Conclusion}

The extreme flexibility of human behavior, even at very short time scales, represents a serious obstacle to the progress of cognitive science. Theoretically, this flexibility leads to an architecture-strategy credit assignment problem that makes it difficult to discern the nature of fixed processing invariants. Empirically, this flexibility presents a serious challenge to devising experiments that exert sufficient control over strategic variation. We have argued that one way to make progress in the face of these challenges is to turn the remarkably adaptive nature of the human system to our advantage in theoretical analysis by using an assumption of optimal adaptation to a utility function given the constraints on information processing. This assumption of cognitively bounded rationality provides a principled basis for sharply narrowing the space of possible strategies and thereby the theoretical predictions. The resulting approach emphasizes the range of possible predictions made from the set of strategies that are optimal given constraints, and thus shifts the primary unit of analysis away from singleton strategies to spaces of strategies, and the relationship of those spaces to task payoffs. In doing so, we believe it provides another route to "getting close" to the architecture (Newell, 1990) — through understanding how the fixed structure of cognition, perception, and action leaves a discernible mark on behavior by way of adaptation, not in spite of it.

\section{References}

Altmann, E. M., \& Gray, W. D. (2008). An integrated model of cognitive control in task switching. Psychological Review, 115, 602-639.

Anderson, J. R. (1978). Arguments concerning representations for mental imagery. Psychological Review, 85, 249-277.

Anderson, J. R. (1983). The architecture of cognition. Cambridge, MA: Harvard University Press.

Anderson, J. R. (1990). Rational analysis. Hillsdale, NJ: Erlbaum.

Anderson, J. R. (1993). Rules of the mind. Hillsdale, NJ: Erlbaum.

Anderson, J. R. (2007). How can the human mind occur in the physical universe? New York: Oxford University Press.

Anderson, J. R., Bothell, D., Byrne, M. D., Douglass, S., Lebiere, C., \& Qin, Y. (2004). An integrated theory of the mind. Psychological Review, 111, 1036-1060.

Anderson, J. R., \& Lebiere, C. (1998). The atomic components of thought. Mahwah, NJ: Erlbaum.

Anderson, J. R., \& Milson, R. (1989). Human memory: An adaptive perspective. Psychological Review, 96, 703-719.

Anderson, J. R., \& Schooler, L. J. (1991). Reflections of the environment in memory. Psychological Science, 2, 396-408.

Anderson, J. R., Taatgen, N. A., \& Byrne, M. D. (2005). Learning to achieve perfect time sharing: Architectural implications of Hazeltine, Teague, and Ivry (2002). Journal of Experimental Psychology: Human Perception and Performance, 31, 749-761.

Berthier, N. E., Rosenstein, M. T., \& Barto, A. G. (2005). Approximate 
optimal control as a model for motor learning. Psychological Review, 112, 329-346.

Bogacz, R., Brown, E., Moehlis, J., Holmes, P., \& Cohen, J. D. (2006). The physics of optimal decision making: A formal analysis of models of performance in two-alternative forced-choice tasks. Psychological Review, 113, 700-765.

Brumby, D. P., Howes, A., \& Salvucci, D. D. (2007). A cognitive constraint model of dual-task trade-offs in a highly dynamic driving task. In S. J. Payne \& B. Begole (Eds.), Proceedings of the SIGCHI Conference on Human Factors in Computing Systems, CHI' 2007 (pp. 233-242). New York, ACM Press.

Byrne, M. D., \& Anderson, J. R. (2001). Serial modules in parallel: The psychological refractory period and perfect time sharing. Psychological Review, 108, 847-869.

Chater, N., \& Oaksford, M. (1999). Ten years of the rational analysis of cognition. Trends in Cognitive Science, 3, 57-65.

Cohen, J. D., McClure, S. M., \& Yu, A. J. (2007). Should I stay or should I go? Exploration versus exploitation. Philosophical Transactions of the Royal Society B: Biological Sciences, 362, 933-942.

Eng, K., Lewis, R. L., Tollinger, I., Chu, A., Howes, A., \& Vera, A. (2006). Generating automated predictions of behavior strategically adapted to specific performance objectives. Proceedings of ACM Conference on Human Factors in Computing Systems, CHI'O6 (pp. 621-630). Montreal, Quebec, Canada: ACM Press.

Forster, K. I. (1979). Levels of processing and the structure of the language processor. In W. E. Cooper \& E. C. T. Walker (Eds.), Sentence processing: Psycholinguistic studies presented to Merrill Garrett (pp. $27-$ 85). Hillsdale, NJ: Erlbaum.

Fu, W. T., \& Gray, W. D. (2006). Suboptimal tradeoffs in informationseeking. Cognitive Psychology, 52, 195-242.

Geisler, W. S. (2003). Ideal observer analysis. In L. Chalupa \& J. Werner (Eds.), The visual neurosciences (pp. 825-837). Boston: MIT Press.

Gelman, A., \& Hill, J. (2007). Data analysis using regression and multilevel/hierarchical models. New York: Cambridge University Press.

Gigerenzer, G., \& Todd, P. M. (1999). Simple heuristics that make us smart. New York: Oxford University Press.

Goeree, J. K., \& Holt, C. A. (2001). Ten little treasures of game theory and ten intuitive contradictions. American Economic Review, 91, 14021422.

Gray, W. D., \& Boehm-Davis, D. A. (2000). Milliseconds matter: An introduction to microstrategies and to their use in describing and predicting interactive behavior. Journal of Experimental Psychology: Applied, 6, 322-335.

Gray, W. D., John, B. E., \& Atwood, M. E. (1993). Project Ernestine: Validating a GOMS analysis for predicting and explaining real-world performance. Human-Computer Interaction, 8, 237-309.

Gray, W. D., Sims, C. R., Fu, W.-T., \& Schoelles, M. J. (2006). The soft constraints hypothesis: A rational analysis approach to resource allocation for interactive behavior. Psychological Review, 113, 461-482.

Green, D. M., \& Swets, J. A. (1966). Signal detection theory and psychophysics. New York: Wiley.

Hansberger, J. T., Schunn, C. D., \& Holt, R. W. (2006). Strategy variability: How too much of a good thing can hurt performance. Memory \& Cognition, 34, 1652-1666.

Harding, S. G. (1976). Can theories be refuted: Essays on the DuhemQuine thesis. Dordrecht, the Netherlands: Reidel.

Hawkins, H. L., Rodriquez, E., \& Reicher, G. M. (1979). Is time sharing a general ability? (ONR Tech. Rep. No. 3). Eugene: University of Oregon.

Hazeltine, E., Teague, D., \& Ivry, R. B. (2002). Simultaneous dual-task performance reveals parallel response selection after practice. Journal of Experimental Psychology: Human Perception and Performance, 28, $527-545$.
Howes, A., Lewis, R. L., \& Vera, A. (2007). Bounding rational analysis: Constraints on asymptotic performance. In W. D. Gray (Ed.), Integrated models of cognitive systems (pp. 403-413). New York: Oxford University Press.

Howes, A., Lewis, R. L., Vera, A., \& Richardson, J. (2005). InformationRequirements Grammar: A theory of the structure of competence for interaction. In Proceedings of the 27th Annual Meeting of the Cognitive Science Society. Mahwah, NJ: Erlbaum.

Howes, A., Vera, A., Lewis, R. L., \& McCurdy, M. (2004). Cognitive constraint modeling: A formal approach to reasoning about behavior. In K. D. Forbus, D. Gentner, \& T. Regier (Eds.), 26th Annual Meeting of the Cognitive Science Society, CogSci2004 (pp. 595-600). Hillsdale, NJ: Erlbaum.

Howes, A., \& Young, R. M. (1997). The role of cognitive architecture in modeling the user: Soar's learning mechanism. Human-Computer Interaction, 12, 311-343.

Keppel, G., \& Underwood, B. J. (1962). Proactive inhibition in short-term retention of single items. Journal of Verbal Learning and Verbal Behavior, 1, 153-161.

Kieras, D. E., \& Meyer, D. E. (2000). The role of cognitive task analysis in the application of predictive models of human performance. In J. M. Schraagen, S. F. Chipman, \& V. L. Shalin (Eds.), Cognitive task analysis (pp. 237-260). Mahwah, NJ: Erlbaum.

Klapp, S. T., Nelson, J. M., \& Jagacinski, R. J. (1998). Can people tap concurrent bimanual rhythms independently? Journal of Motor Behavior, 30, 301-322.

Levy, J., \& Pashler, H. (2001). Is dual-task slowing instruction dependent? Journal of Experimental Psychology: Human Perception and Performance, 27, 862-869.

Lewis, R. L., Newell, A., \& Polk, T. A. (1989). Toward a Soar theory of taking instructions for immediate reasoning tasks. In Proceedings of the Eleventh Annual Conference of the Cognitive Science Society (pp. 514521). Hillsdale, NJ: Erlbaum.

Lewis, R. L., Vera, A., \& Howes, A. (2004). A constraint-based approach to understanding the composition of skill. In M. Lovett, C. Schunn, C. Lebiere, \& P. Munro (Eds.), Proceedings of the Sixth International Conference on Cognitive Modeling, ICCM'04 (pp. 148-153). Mahwah, NJ: Erlbaum.

Lindstrom, M. J., \& Bates, D. M. (1990). Nonlinear mixed effects models for repeated measures data. Biometrics, 46, 673-687.

Lloyd, E. A. (1999). Evolutionary psychology: The burden of proof. Biology and Philosophy, 14, 211-233.

Lohse, G. L., \& Johnson, E. J. (1996). A comparison of two process tracing methods for choice tasks. Organizational Behavior and Human Decision Processes, 68, 28-43.

Lovett, M. C., \& Anderson, J. R. (1996). History of success and current context in problem solving: Combined influences on operator selection. Cognitive Psychology, 31, 168-217.

Maloney, L. T., Trommershäuser, J., \& Landy, M. S. (2007). Questions without words: A comparison between decision making under risk and movement planning under risk. In W. Gray (Ed.), Integrated models of cognitive systems (pp. 297-313). New York: Oxford University Press.

McCann, R. S., \& Johnston, J. C. (1992). Locus of the single-channel bottleneck in dual-task interference, Journal of Experimental Psychology: Human Perception and Performance, 18, 471-484.

Meyer, D. E., Abrams, R. A., Kornblum, S., Wright, C. E., \& Smith, J. E. K. (1988). Optimality in human motor performance: Ideal control of rapid aimed movements. Psychological Review, 95, 340-370.

Meyer, D. E., \& Kieras, D. E. (1997a). A computational theory of executive cognitive processes and multiple-task performance: Part 1. Basic mechanisms. Psychological Review, 104, 3-65.

Meyer, D. E., \& Kieras, D. E. (1997b). A computational theory of executive control processes and human multiple-task performance: Part 2. 
Accounts of psychological refractory-period phenomena. Psychological Review, 104, 749-791.

Meyer, D. E., \& Kieras, D. E. (1999). Précis to a practical unified theory of cognition and action: Some lessons from computational modeling of human multiple-task performance. In D. Gopher \& A. Koriat (Eds.), Attention and performance XVII: Cognitive regulation of performance: Interaction of theory and application (pp. 17-88). Cambridge, MA: MIT Press.

Newell, A. (1973). You can't play 20 questions with nature and win: Projective comments on papers in this symposium. In W. G. Chase (Ed.), Visual information processing (pp. 283-310). New York: Academic Press.

Newell, A. (1990). Unified theories of cognition. Cambridge, MA: Harvard University Press.

Newell, A., \& Simon, H. A. (1972). Human problem solving. Englewood Cliffs, NJ: Prentice Hall

Newell, A., \& Rosenbloom, P. S. (1981). Mechanisms of skill acquisition and the law of practice. In J. R. Anderson (Ed.), Cognitive skills and their acquisition (pp. 1-51). Hillsdale, NJ: Erlbaum.

Oaksford, M., \& Chater, N. (1994). A rational analysis of the selection task as optimal data selection. Psychological Review, 101, 608-631.

Ohlsson, S. (1996). Learning from performance errors. Psychological Review, 103, 241-262.

Pashler, H. (1998). The psychology of attention. Cambridge, MA: MIT Press.

Payne, S. J., Howes, A., \& Reader, W. R. (2001). Adaptively distributing cognition: A decision-making perspective on human-computer interaction. Behavior and Information Technology, 20, 5, 339-346.

Payne, S. J., Richardson, J., \& Howes, A. (2000). Strategic use of familiarity in display-based problem solving. Journal of Experimental Psychology: Learning, Memory, and Cognition, 26, 1685-1701.

Pinheiro, J., Bates, D., DebRoy, S., Sarkar, D., \& the R Core team. (2009). nlme: Linear and nonlinear mixed effects models (Version 3.1-92) [Computer software]. Retrieved from http://cran.r-project.org/web/ packages/nlme/index.html

Pitt, M. A., Kim, W., Navarro, D. J., \& Myung, J. I. (2006). Global model analysis by parameter space partitioning. Psychological Review, 113, 57-83

Pitt, M. A., Myung, I. J., \& Zhang, S. (2002). Toward a method of selecting among computational models of cognition. Psychological Review, 109, 472-491.

Pylyshyn, Z. W. (1973). What the mind's eye tells the mind's brain: A critique of mental imagery. Psychological Bulletin, 80, 1-24.

Reichle, E. D., \& Laurent, P. A. (2006). Using reinforcement learning to understand the emergence of "intelligent" eye-movement behavior during reading. Psychological Review, 113, 390-408.

Roberts, S., \& Pashler, H. (2000). How persuasive is a good fit? A comment on theory testing. Psychological Review, 107, 358-367.

Ruthruff, E., Pashler, H. E., \& Klaassen, A. (2001). Processing bottlenecks in dual-task performance: Structural limitation or voluntary postponement? Psychonomic Bulletin \& Review, 8, 73-80.

Schumacher, E. H., Lauber, E. J., Glass, J. M., Zurbriggen, E. L., Gmeindl, L., Kieras, D. E., \& Meyer, D. E. (1999). Concurrent response-selection processes in dual-task performance: Evidence for adaptive executive control of task scheduling. Journal of Experimental Psychology: Human Perception and Performance, 25, 791-814.

Schumacher, E. H., Seymour, T. L., Glass, J. M., Fencsik, D. E., Lauber, E. J., Kieras, D. E., \& Meyer, D. E. (2001). Virtually perfect time sharing in dual-task performance: Uncorking the central cognitive bottleneck. Psychological Science, 12, 101-108.

Schunn, C. D., \& Reder, L. M. (2001). Another source of individual differences: Strategy adaptivity to changing rates of success. Journal of Experimental Psychology: General, 130, 59-76.
Schweickert, R. (1980, August 8). Critical-path scheduling of mental processes in a dual task. Science, 209, 704-706.

Schweickert, R., Fisher, D. L., \& Proctor, R. W. (2003). Steps toward building mathematical and computer models from cognitive task analyses. Human Factors, 45, 77-103.

Shanks, D. R., Tunney, R. J., \& McCarthy, J. D. (2002). A re-examination of probability matching and rational choice. Journal of Behavioral Decision Making, 15, 233-250.

Siegler, R. S. (1994). Cognitive variability: A key to understanding cognitive development. Current Directions in Psychological Science, 3, $1-5$.

Siegler, R. S. (1999). Strategic development. Trends in Cognitive Development, 3, 430-435.

Simon, H. A. (1955). A behavioral model of rational choice. Quarterly Journal of Economics, 69, 99-118.

Simon, H. A. (1957). Models of man. New York: Wiley.

Simon, H. A. (1979). Rational decision making in business organizations. The American Economic Review, 69, 493-513.

Simon, H. A. (1989). Models of thought. New Haven, CT: Yale University Press.

Simon, H. A. (1991). Cognitive architectures and rational analysis: Comment. In K. VanLehn (Ed.), Architectures for intelligence: The 22nd Carnegie Mellon Symposium on Cognition (pp. 25-39). Hillsdale, NJ: Erlbaum.

Simon, H. A. (1992). What is an "explanation" of behavior? Psychological Science, 3, 150-161.

Simon, H. A. (1997). Models of bounded rationality: Vol. 3. Empirically grounded economic reason. Cambridge, MA: MIT Press.

Smith, M. R., Lewis, R. L., Howes, A., Chu, A., \& Green, C. (2008). More than 8,192 ways to skin a cat: Modeling behavior in multidimensional strategy spaces. In B. C. Love, K. McRae, \& V. M. Sloutsky (Eds.), Proceedings of the 30th Annual Conference of the Cognitive Science Society (pp. 1441-1446). Austin, TX: Cognitive Science Society.

Sperling, G., \& Dosher, B. A. (1986). Strategy optimization in human information processing. In K. R. Boff, L. Kaufman, \& J. P. Thomas (Eds.), Handbook of perception and human performance: Vol. 1. Sensory processes and perception (pp. 1-65). New York: Wiley.

Sutton, R. S., \& Barto, A. G. (1998). Reinforcement learning: An introduction. Cambridge, MA: MIT Press.

Swets, J. A., Tanner, W. P. J., \& Birdsall, T. G. (1961). Decision processes in perception. Psychological Review, 68, 301-340.

Taatgen, N. A. (2005). Modeling parallelization and speed improvement in skill acquisition: From dual tasks to complex dynamic skills. Cognitive Science, 29, 421-455.

Tanner, W. P. J., \& Swets, J. A. (1954). A decision-making theory of visual detection. Psychological Review, 61, 401-409.

Tenenbaum, J. B., Griffiths, T. L., \& Kemp, K. (2006). Theory-based Bayesian models of inductive learning and reasoning. Trends in Cognitive Science, 10, 309-318.

Tollinger, I., Lewis, R. L., McCurdy, M., Tollinger, P., Vera, A., Howes, A., \& Pelton, L. (2005). Supporting efficient development of cognitive models at multiple skill levels: Exploring recent advances in constraintbased modeling. In Proceedings of the ACM Conference on Human Factors and Computing Systems, CHI'05 (pp. 411-420). Portland, OR: ACM Press.

Trommershäuser, J., Maloney, L. T., \& Landy, M. S. (2003a). Statistical decision theory and rapid, goal-directed movements. Journal of the Optical Society of America A: Optics, Image Science \& Vision, 20, $1419-1433$

Trommershäuser, J., Maloney, L. T., \& Landy, M. S. (2003b). Statistical decision theory and tradeoffs in motor response. Spatial Vision, 16, 255-275.

Van Zandt, T., \& Ratcliff, R. (1995). Statistical mimicking of reaction time 
data: Single process models, parameter variability, and mixtures. Psychonomic Bulletin \& Review, 2, 20-54.

Vera, A., Tollinger, I., Eng, K., Lewis, R., \& Howes, A. (2005). Architectural building blocks as the locus of adaptive behavior selection. In B. G. Bara, L. Barsalou, \& M. Bucciarelli (Eds.), Proceedings of the 27th Annual Meeting of the Cognitive Science Society (pp. 2295-2301). Mahwah, NJ: Erlbaum.

Vera, A. H., Howes, A., McCurdy, M., \& Lewis, R. L. (2004). A constraint satisfaction approach to predicting skilled interactive performance. In CHI'04: Proceedings of the SIGCHI Conference on Human Factors in Computing Systems (pp. 121-128). New York: ACM Press.

Wason, P. (1966). Reasoning. In B. M. Foss (Ed.), New horizons in psychology (pp. 135-151). Hammondsworth, United Kingdom: Penguin.

Weisstein, E. W. (2008). Gamma distribution. Retrieved from http:// mathworld.wolfram.com/GammaDistribution.html

\section{Appendix}

Details of the Analysis of the ACT-R Psychological Refractory Period Models

\section{Architectural Assumptions}

We made use of a single level of activation noise for all models (the same value used in Byrne and Anderson's, 2001, models of Experiment 3 and Experiment 4, though not in E1 and E2). Following Byrne and Anderson (2001), we assumed that a retrieval process was required for Task 2 only. Also following Byrne and Anderson, we assumed that the motor system was always "unprepared" for the Task 1 punch response but prepared for Task 2 . Response preparation therefore took $150 \mathrm{~ms}$ for Task 1 but only $100 \mathrm{~ms}$ for Task 2. Byrne and Anderson assumed Task 1 took 150 $\mathrm{ms}$ in their models of Experiments 1, 3, and 4 but $50 \mathrm{~ms}$ less in their model of Experiment 2. To compensate for the additional 50 ms taken by the motor system in our model of Experiment 2, we reduced the time required by perception so as to fit the Task 1 response at 1,000 ms. Following Byrne and Anderson, we assumed that the motor system processes were subject to uniform noise at a level that was consistent for all four experiments.

All values of easy and hard Task 2 base level activation were chosen so as to fit Task 2 response time at 1,000-ms SOA, although we generated slightly different values than those settled on by Byrne and Anderson (2001). Our analysis indicated that the 100-200 trials used by Byrne and Anderson were not sufficient to get a tight estimate of the mean performance of the model relative to the variability in the human data. ${ }^{\mathrm{A} 1}$ We used more trials $(2,000)$, and as a consequence different activation values emerged to obtain the best fit.

\section{Strategy Assumptions}

For example, Strategy 10 is a strategy in which after the Task 1 motor prepare process has issued a completion control signal
(Prep), an unlock process releases Task 2 completion (the Explicit unlock process column), and then Task 2 attend is processed (Task 2 attend deferment column). See Table A1.

Table A1

A Description of the Features of Each of the 12 Strategies Used to Test the ACT-R Account of Experiments 1-4

\begin{tabular}{rlll}
\hline $\begin{array}{c}\text { Strategy } \\
\text { no. }\end{array}$ & $\begin{array}{c}\text { Completion control } \\
\text { signal }\end{array}$ & $\begin{array}{c}\text { Explicit unlock } \\
\text { process }\end{array}$ & $\begin{array}{c}\text { Task 2 attend } \\
\text { deferment }\end{array}$ \\
\hline 1 & Prep & No & No \\
2 & Prep & No & Yes \\
3 & Process & No & No \\
4 & Process & No & Yes \\
5 & Prep & Defer & No \\
6 & Prep & Defer & Yes \\
7 & Process & Defer & No \\
8 & Process & Defer & Yes \\
9 & Prep & Unlock & No \\
10 & Prep & Unlock & Yes \\
11 & Process & Unlock & No \\
12 & Process & & Yes \\
\hline
\end{tabular}

${ }^{\text {A1 }}$ More specifically, in some cases the $95 \%$ confidence interval around the mean model predictions approached the size of the empirical effects of interest.
Received September 1, 2007

Revision received June 29, 2009

Accepted June 30, 2009 